\title{
Diffusion in systems with static disorder
}

\author{
P. J. H. Denteneer and M. H. Ernst \\ Institute for Theoretical Physics, Princetonplein 5, P.O. Box 80006, 3508-TA Utrecht, The Netherlands
}

(Received 28 June 1983)

\begin{abstract}
We study diffusion in systems with static disorder, characterized by random transition rates $\left\{w_{n}\right\}$, which may be assigned to the bonds [random-barrier model (RBM)] or to the sites [randomjump-rate model (RJM)]. We make an expansion in powers of the fluctuations $\delta_{n}=\left(w_{n}^{-1}-\left\langle w^{-1}\right\rangle\right) /$ $\left\langle w^{-1}\right\rangle$ around the exact diffusion coefficient $D=1 /\left\langle w^{-1}\right\rangle$ in the low-frequency regime, using diagrammatic methods. For the one-dimensional models we obtain a systematic expansion in powers of $\sqrt{z}$ of the response function (transport properties) and Green's function (spectral properties). The frequency-dependent diffusion coefficient in the RBM is found as $U_{0}(z)=D-\frac{1}{2} \kappa_{2} \sqrt{D z}$ $+\alpha_{0} z+\alpha_{1} z^{3 / 2}+\cdots$, where $\kappa_{2}=\left\langle\delta^{2}\right\rangle, \alpha_{0}$ includes up to fourth-order fluctuations and $\alpha_{1}$ up to sixth order. In the RJM, $U_{0}(z)=D$. Similarly, we obtain results (very different in RBM and RJM) for the frequency-dependent Burnett coefficient $U_{2}(z)$ and the single-site Green's function $\hat{G}_{0}(z)$ [which determines the density of eigenstates $\mathscr{N}(\epsilon)$ and the inverse localization length $\gamma(\epsilon)$ of relaxational modes of the system]. The spectral properties of both models are identical and agree with exact results at low frequencies for the spectral properties of random harmonic chains. The long-time behavior of the velocity autocorrelation function in $\operatorname{RBM}$ is $\varphi_{2}(t) \simeq(\cdots) t^{-3 / 2}+(\cdots) t^{-5 / 2}$ and for the Burnett correlation function $\varphi_{4}(t) \simeq(\cdots) t^{-3 / 2}$, with coefficients that vanish on a uniform lattice. For the RJM, $\varphi_{2}(t)=D \delta_{+}(t)$ and $\varphi_{4}(t) \simeq(\cdots) t^{-1 / 2}$. The long-time behavior of the moments of displacement $\left\langle n^{2}\right\rangle_{t}$ and $\left\langle n^{4}\right\rangle_{t}$ and the staying probability $P_{0}(t)$ are calculated up to relative order $t^{-3 / 2}$. A comparison of our exact results with those of the effective-medium (or hypernettedchain) approximation (EMA) shows that the coefficient $\alpha_{0}$ in $U_{0}(z)$ as given by EMA is incorrect, contrary to suggestions made in the literature. For the RJM all results can be trivially extended to higher-dimensional systems.
\end{abstract}

\section{INTRODUCTION}

Diffusion or hopping conductivity in systems with static disorder shows interesting non-Markovian behavior, which recently received much attention. ${ }^{1-16} \mathrm{~A}$ review of hopping models was given by Alexander et al. ${ }^{1}$ and more recent results can be found in Refs. $2-16$. One can assign the static disorder to the bonds, as is done in randombarrier models $1-3,6,12,17,18$ or to the sites as is done in random-jump-rate models. ${ }^{7,14}$ Another class of models are exactly solvable one-dimensional stochastic Lorentz models, discussed by van Beijeren, ${ }^{15,19}$ in which the lattice distances are random variables.

Here we restrict ourselves to hopping models of the random-barrier or random-jump-rate type, described by a master equation. It is known from approximate $^{1,2,4,8,10,17,20}$ and exact ${ }^{12}$ theories that the frequencydependent diffusion coefficient $U_{0}(z)$ at low frequencies $(z \rightarrow 0)$ behaves as $U_{0}(z)=D-\frac{1}{2} \kappa_{2} \sqrt{D z}+\cdots$, where $D=1 /\left\langle w^{-1}\right\rangle$ is the exact diffusion coefficient and $\kappa_{2}=\left\langle\left(w^{-1}-\left\langle w^{-1}\right\rangle\right)^{2}\right\rangle /\left\langle w^{-1}\right\rangle^{2}$. The inverse Laplace transform of $U_{0}(z)$ corresponds to the velocity autocorrelation function (VACF), $\varphi_{2}(t)$, which shows the wellknown long-time tail $\varphi_{2}(t) \sim t^{-(d+2) / 2}$ of diffusion in stationary random media, such as in the $d$-dimensional Lorentz gas. ${ }^{21}$ For higher-order terms, only approximate results based on an effective-medium approximation (EMA) have been obtained by Webman and Klafter ${ }^{10}$ with terms of order up to $z$ included and by Haus et al. ${ }^{8}$ with terms of order up to $z^{3 / 2}$ included. It has been suggest$\mathrm{ed}^{8,10}$ that the EMA gives exact results to terms of order $z$ included.

In this paper we present new results for the asymptotic (low-frequency and long-time) properties of hopping models of random-barrier or random-jump-rate type, which are based on an expansion in powers of the fluctuations $\delta_{n}=\left(w^{-1}-\left\langle w^{-1}\right\rangle\right) /\left\langle w^{-1}\right\rangle$ around the exact diffusion coefficient (see Sec. III). The terms in this expansion are calculated using a diagrammatic method (see Appendix $\mathrm{A}$ ) and it is shown that this fluctuation expansion is a systematic expansion in powers of $\sqrt{z}$ (see Appendix B). In Sec. IV we give explicit results for the frequencydependent diffusion coefficient in the form $U_{0}(z)=D$ $-\frac{1}{2} \kappa_{2} \sqrt{D z}+\alpha_{0} z+\alpha_{1} z^{3 / 2}+\cdots$, and for many related functions of interest. Our calculations show that the EMA is in fact the hypernetted-chain approximation ${ }^{22,23}$ for the present diagrammatic method and that the EMA results for $U_{0}(z)$ are already incorrect in terms of order $z$, in disagreement with the suggestions of Webman and Klafter and Haus et al. (see Sec. IV). We have also calculated in Sec. IV the staying probability, $P_{0}(t)$, and the single-site Green's function $\hat{G}_{0}(z)$, which determines the spectral properties of the eigenmodes of the system. The resulting eigenvalue problem is identical to that for the harmonic chain with random masses or random force constants. Our results appear to be in complete agreement with re- 
cent exact results of Nieuwenhuizen ${ }^{24}$ for the density of states and inverse localization length of eigenfunctions in a harmonic chain with random masses.

For a random-jump-rate model, discussed in Refs. 7 and 25 , all results can be trivially extended to higherdimensional systems, as shown in Sec. V, where some of the $d$-dimensional lattice functions are analyzed. In Sec. VI we present a brief discussion of our results.

The hopping models of interest are described by a master equation of the general form

$$
\dot{p}_{n}=-\sum_{m} L_{n m} p_{m}=-(L p)_{n},
$$

where $p_{n}(t)$ represents the probability of finding the random walker (hopping particle) at time $t$ on site $n$ $(n=0,1,2, \ldots, N-1)$ of a regular one-dimensional lattice with periodic boundary conditions $\left(p_{n+N}=p_{n}\right)$ and lattice distance equal to unity (an overdot denotes a derivative with respect to time). The transition matrix $L_{n m}$ allows only transitions between nearest neighbors and the transition rates $w_{n}(n=0,1, \ldots, N-1)$ occurring in $L_{n m}$ are positive time-dependent random variables, with a siteindependent distribution $\pi(w)$. The first few inverse moments $\left\langle w^{-l}\right\rangle=\int_{0}^{\infty} w^{-l} \pi(w) d w$ are assumed to exist $(l=1,2, \ldots, 6)$. In this paper angular brackets \langle\rangle always denote an average over random variables $\left\{w_{n}\right\}$.

We consider two types of hopping models, a bond problem and a site problem, in which the random variables are assigned to the bonds [random-barrier model (RBM)] and to the sites [random-jump-rate model (RJM)], respectively. The master equation for the RBM has the explicit form

$$
\begin{aligned}
\dot{p}_{n} & =w_{n-1}\left(p_{n-1}-p_{n}\right)+w_{n}\left(p_{n+1}-p_{n}\right) \\
& =-\left(E_{n}^{-1}-1\right) w_{n}\left(E_{n}-1\right) p_{n},
\end{aligned}
$$

where $E_{n} f_{n}=f_{n+1}$ and $w_{n}$ denotes the random transition rate for jumps across the nearest-neighbor bond between the pair $(n, n+1)$. The stationary solution of $(1.2)$ is a site-independent constant:

$$
\psi_{n}=1 \text {, }
$$

normalized such that $\sum_{n=0}^{N-1} \psi_{n}=N$.

The master equation for the (isotropic) RJM reads

$$
\begin{aligned}
\dot{p}_{n} & =w_{n-1} p_{n-1}-2 w_{n} p_{n}+w_{n+1} p_{n+1} \\
& =-\left(2-E_{n}-E_{n}^{-1}\right) w_{n} p_{n} .
\end{aligned}
$$

Here a random jump rate $v_{n}$ (or waiting time $1 / v_{n}$ ) is assigned to the $n$th site and the particle jumps with equal probability to one of its nearest-neighbor sites. Hence the transition rate for a jump to any nearest neighbor of the $n$th site is $w_{n}=\frac{1}{2} v_{n}$. The stationary solution of (1.4) is $\psi_{n}=C / w_{n}$, where $C$ is determined from the normalization condition $\sum_{n} \psi_{n}=N$, yielding $C^{-1}=\sum_{n}\left(N w_{n}\right)^{-1}$ $=\left\langle w^{-1}\right\rangle$. Using the exact diffusion coefficient for this model $D=1 /\left\langle w^{-1}\right\rangle$, as given by Haus et al. ${ }^{7}$ one finds the stationary solution of (1.4) as

$$
\psi_{n}=D / w_{n} .
$$

Haus et al. further showed that $\frac{1}{2}(d / d t)\left\langle n^{2}\right\rangle_{t}=D$ is an exact relation in the RJM for all times and for general dimensionality, implying that the VACF $\varphi_{2}(t)=D \delta_{+}(t)$ [with $\int_{0}^{\infty} \delta_{+}(t) d t=1$ ], without any long-time tail. However, long-time tails show up in the Burnett correlation function, related to the fourth moment of the displacement. ${ }^{20,26}$

\section{PROBABILITIES OF DISPLACEMENT, RESPONSE AND GREEN'S FUNCTIONS}

From a macroscopic point of view one is interested in $P_{n}(t)$, the probabilities of displacements $n$ at time $t$ in a stationary initial ensemble, averaged over the random variables $\left\{w_{n}\right\}$ [ the analog of the Van Hove function $G(r, t)$ ], which determines most quantities of physical interest, such as the transport properties. Of particular interest are the lower moments

$$
\left\langle n^{l}\right\rangle_{t}=\sum_{n=0}^{N-1} n^{l} P_{n}(t) .
$$

For instance the diffusion coefficient follows from the long-time behavior of the mean-square displacement, $\left\langle n^{2}\right\rangle_{t} \simeq 2 D t$.

The Fourier transform of $P_{n}(t)$ (the so-called intermediate scattering function) is the generating function for these moments. In our analysis we actually calculate the response function $\mathscr{F}(q, z)$, which is the Fourier-Laplace transform of $P_{n}(t)$ :

$$
\mathscr{F}(q, z)=\sum_{n=0}^{N-1} e^{i q n} \hat{P}_{n}(z)
$$

Laplace transforms are in general denoted as $\hat{\boldsymbol{F}}(z)$ $=\int_{0}^{\infty} e^{-z t} F(t) d t$ and the reciprocal lattice vector $q=2 \pi l / N$ with $l=-\frac{1}{2} N+1, \ldots, \frac{1}{2} N$ lies in the first Brillouin zone (1BZ). It is further convenient to use the orthogonality relations

$$
N^{-1} \sum_{n=0}^{N-1} e^{i n\left(q-q^{\prime}\right)}=\delta_{q q^{\prime}}, \quad N^{-1} \sum_{q \in 1 \mathrm{BZ}} e^{i q(n-m)}=\delta_{n m} \text {. }
$$

Henceforward the limits on the summation signs will be dropped and every sum over sites runs from $n=0$ until $n=N-1$ and all $q$ sums run over the $1 \mathrm{BZ}$.

In generalized hydrodynamics it is often convenient to express the response function $\mathscr{F}(q, z)$ in terms of a generalized diffusion coefficient $U(q, z)$ as

$$
\mathcal{F}(q, z)=\left[z+q^{2} U(q, z)\right]^{-1} .
$$

The ordinary diffusion coefficient $D$ follows from

$$
D=\lim _{z \rightarrow 0} \lim _{q \rightarrow 0} U(q, z) .
$$

The Laplace transforms of the moments of displacement, $\left\langle n^{l}\right\rangle(z)$, generated by (2.2), i.e.,

$\mathscr{F}(q, z)=\frac{1}{z}-\frac{1}{2} q^{2}\left\langle n^{2}\right\rangle(z)+\frac{1}{4 !} q^{4}\left\langle n^{4}\right\rangle(z)+\cdots$,

can be expressed in terms of $U(q, z)$. To do so we expand

$$
U(q, z)=U_{0}(z)-q^{2} U_{2}(z)+\cdots
$$


and use it to obtain a similar expression for $\mathscr{F}(q, z)$. Comparison with (2.6) then yields

$$
\begin{aligned}
& \frac{1}{2}\left\langle n^{2}\right\rangle(z)=z^{-2} U_{0}(z), \\
& \frac{1}{4 !}\left\langle n^{4}\right\rangle(z)=z^{-2} U_{2}(z)+z^{-3} U_{0}^{2}(z) .
\end{aligned}
$$

Here $U_{0}(z)$ is the "frequency-dependent" diffusion coefficient $\left[U_{0}(0)=D\right]$ and $U_{2}(z)$ is the frequency-dependent modified Burnett coefficient. ${ }^{27}$ The inverse Laplace transforms of $U_{0}(z)$ and $U_{2}(z)$ will be referred to as the velocity autocorrelation function (VACF) $\varphi_{2}(t)$ and the Burnett correlation function $\varphi_{4}(t)$, respectively. ${ }^{19,26,27}$

In order to relate the macroscopic probability of displacements to the solution of the master equation (1.1), we observe that the conditional probability or Green'sfunction solution $\Gamma_{n m}(t)$ of (1.1) with $\Gamma_{n m}(0)=\delta_{n m}$ equals the probability of a displacement $(n-m)$ in a frozen configuration $\left\{w_{n}\right\}$, given that the walker starts at site $m$. Let $\psi_{m}$ be the stationary initial distribution (with normalization $\left.\sum_{m} \psi_{m}=N\right)$ for a frozen configuration $\left\{w_{n}\right\}$, as given in (1.3) and (1.5); then

$$
\pi_{n}(t)=\sum_{m} \Gamma_{n+m, m}(t) \psi_{m} / N
$$

is the probability of a displacement $n$ in a frozen configuration $\left\{w_{n}\right\}$ averaged over all possible starting positions of the random walker. The macroscopic probability of displacements is then

$$
P_{n}(t)=\left\langle\pi_{n}(t)\right\rangle=N^{-1} \sum_{m}\left\langle\Gamma_{n+m, m}(t) \psi_{m}\right\rangle,
$$

where the angular brackets denote an average over the random variables $\left\{w_{n}\right\}$.

The probabilities $\pi_{n}(t)$ for a frozen set of $\left\{w_{n}\right\}$ values do not have the translational symmetry of the lattice; however, averages \langle\rangle do have this symmetry. We therefore introduce the Fourier transform

$$
\rho_{q}(t)=\sum_{n} e^{i q n} \pi_{n}(t) .
$$

With the help of (2.9) and the orthogonality relation (2.3) it can be written in the form

$$
\rho_{q}(t)=\sum_{q^{\prime}} \Gamma_{q q^{\prime}}(t) \Psi_{q^{\prime} q}=[\Gamma(t) \Psi]_{q q},
$$

where we have introduced

$$
\begin{aligned}
& \Gamma_{q q^{\prime}}=N^{-1} \sum_{n, m} e^{i q n} \Gamma_{n m} e^{-i q^{\prime} m}, \\
& \Psi_{q q^{\prime}}=N^{-1} \sum_{n} \psi_{n} e^{i n\left(q-q^{\prime}\right)} .
\end{aligned}
$$

Here $\rho_{q}(t)$ satisfies the transformed master equation:

$$
\dot{\rho}_{q}=-\sum_{q^{\prime}} L_{q q^{\prime}} \rho_{q^{\prime}}=-(L \rho)_{q}
$$

The transition matrix in the RBM can be obtained directly from (1.2), (2.11), and its inverse:

$$
\begin{aligned}
L_{q q^{\prime}} & =N^{-1} \sum_{n} e^{i q n}\left(E_{n}^{-1}-1\right) w_{n}\left(E_{n}-1\right) e^{-i q^{\prime} n} \\
& =f^{*}(q) W_{q q^{\prime}} f\left(q^{\prime}\right)=\left(f^{*} W f\right)_{q q^{\prime}}
\end{aligned}
$$

and in the RJM from (1.4):

$$
L_{q q^{\prime}}=f^{*}(q) f(q) W_{q q^{\prime}}=\left(f^{*} f W\right)_{q q^{\prime}},
$$

where

$$
\begin{aligned}
& W_{q q^{\prime}}=N^{-1} \sum_{n} w_{n} e^{i n\left(q-q^{\prime}\right)}, \\
& f(q)=e^{-i q}-1 .
\end{aligned}
$$

The Laplace transformation of (2.12) reads

$$
\hat{\rho}_{q}(z)=\sum_{q^{\prime}} \hat{\Gamma}_{q q^{\prime}}(z) \Psi_{q^{\prime} q}=[\widehat{\Gamma}(z) \Psi]_{q q}
$$

where the Green's function is defined as

$$
\widehat{\Gamma}_{q q^{\prime}}(z)=\left[(z+L)^{-1}\right]_{q q^{\prime}} .
$$

The response function can finally be put in the form

$$
\begin{aligned}
\mathscr{F}(q, z) & =\left\langle\hat{\rho}_{q}(z)\right\rangle=\sum_{q^{\prime}}\left\langle\hat{\Gamma}_{q q^{\prime}}(z) \Psi_{q^{\prime} q}\right\rangle \\
& =\left\langle(z+L)^{-1} \Psi\right\rangle_{q q} .
\end{aligned}
$$

Note that the response function differs in general from the average Green's function (which is the diagonal element of the average of the Green's function $\hat{\Gamma}_{q q^{\prime}}$ ):

$$
\mathscr{G}(q, z)=\left\langle\frac{1}{z+L}\right\rangle_{q q} .
$$

However, in the RBM $\mathscr{F}(q, z)$ and $\mathscr{G}(q, z)$ coincide since $\psi_{m}=1$ is the uniform stationary distribution (1.3) or equivalently $\Psi_{q q^{\prime}}=\delta_{q q^{\prime}}$.

A quantity of interest is the density of eigenstates. If one represents $\rho_{q}(t)$ by $\rho_{q}(t)=e^{-\epsilon(q) t} \rho_{q}$ then (2.12) reduces to an eigenvalue problem, $L \rho=\epsilon \rho$ with solutions $\left\{\epsilon^{\alpha}, \rho_{q}^{(\alpha)}\right\}$ $(\alpha=1,2, \ldots, N)$. Using the well known identity $(x-i 0)^{-1}=\mathrm{P}(1 / x)+\pi i \delta(x)$, where $\mathrm{P}$ denotes the principal value and $\delta(x)$ the Dirac $\delta$ function, we can write the average density of eigenstates per lattice site as

$$
\begin{aligned}
\mathscr{N}(\epsilon) & =N^{-1} \sum_{\alpha}\left\langle\delta\left(\epsilon-\epsilon_{\alpha}\right)\right\rangle \\
& =(\pi N)^{-1} \operatorname{Im}\left[\sum_{\alpha}\left\langle\left(\epsilon-\epsilon_{\alpha}-i 0\right)^{-1}\right\rangle\right] \\
& =-\pi^{-1} \operatorname{Im}\left[N^{-1} \sum_{q}\left\langle(-\epsilon+L+i 0)^{-1}\right\rangle\right] \\
& =-\pi^{-1} \operatorname{Im}\left[\hat{G}_{0}(-\epsilon+i 0)\right] .
\end{aligned}
$$

Here $\hat{G}_{0}(z)$ is the average single-site Green's function defined in terms of (2.21) through

$$
\widehat{G}_{n}(z)=N^{-1} \sum_{q} e^{-i q n} \mathscr{G}(q, z) .
$$

Another quantity of interest related to the single-site 
Green's function is the exponential growth rate (inverse localization length) $\gamma(\epsilon)$ of eigenfunctions. In onedimensional cases this concept ${ }^{28-31}$ can be explained by considering our eigenvalue problem in configuration space by putting $p_{n}(t)=e^{-\epsilon t} p_{n}$ in (1.2) and (1.4). The transformations $u_{n}=\frac{1}{2} w_{n}\left(p_{n+1}-p_{n}\right)$ for $\mathrm{RBM}$ and $u_{n}=w_{n} p_{n}$ for RJM map these eigenvalue equations onto

$$
-\epsilon u_{n}=w_{n}\left(u_{n+1}+u_{n-1}-2 u_{n}\right) \text {. }
$$

In this case we do not use periodic boundary conditions but consider eigenfunctions vanishing at both ends of the chain. The solution $u_{N}(\epsilon)$ of this recursion relation with $u_{-1}=0$ and $u_{0}=1$ is a polynomial of degree $N$ in $\epsilon$, where the coefficient of $(-\epsilon)^{N}$ is given by $\prod_{n=0}^{N-1}\left(1 / w_{n}\right)$. Let $\epsilon_{n}$ with $n=0,1,2, \ldots, N-1$ be the (unknown) zeros of this polynomial, then

$$
u_{N}(\epsilon)=\prod_{n=0}^{N-1}\left[\left(\epsilon_{n}-\epsilon\right) / w_{n}\right]
$$

An eigenfunction must vanish at both ends of the chain. Since $u_{N}\left(\epsilon_{n}\right)=0$, the zeros $\epsilon_{n}$ of $u_{N}(\epsilon)$ are the eigenvalues. If the solutions of (2.24) grow exponentially fast, then the large- $N$ behavior of $\gamma(\epsilon)=N^{-1}\left\langle\ln \left|u_{N}(\epsilon)\right|\right\rangle$ with $\epsilon \neq \epsilon_{n}$ measures the exponential growth rate provided $\gamma(\epsilon)$ is positive. In the thermodynamic limit, where the spectrum becomes dense, $\gamma(\epsilon)$ can be defined by taking $\epsilon$ just above or below the real- $\epsilon$ axis, so that

$$
\begin{aligned}
\gamma(\epsilon) & =\operatorname{Re}\left\{N^{-1}\left\langle\ln \left[u_{N}(\epsilon \pm i 0)\right]\right\rangle\right\} \\
& =\operatorname{Re}\left[N^{-1} \sum_{n=0}^{N-1}\left\langle\ln \left[\left(\epsilon_{n}-\epsilon \pm i 0\right) / w_{n}\right]\right\rangle\right] .
\end{aligned}
$$

The positivity of this function is related to the exponential localization of all eigenfunctions. ${ }^{28,29,31}$

For our purpose it is more convenient to consider $d \gamma / d \epsilon$ as it is directly related to the single-site Green's function (2.23):

$$
\begin{aligned}
\frac{d \gamma}{d \epsilon} & =-\operatorname{Re} N^{-1} \sum_{n=0}^{N-1}\left\langle\frac{1}{\epsilon_{n}-\epsilon \pm i 0}\right\rangle \\
& =-N^{-1} \operatorname{Re} \sum_{q}\left\langle\frac{1}{L-\epsilon \pm i 0}\right\rangle_{q q} \\
& =-\operatorname{Re} \hat{G}_{0}(-\epsilon \pm i 0) .
\end{aligned}
$$

Here $\gamma(0)=0$ since the eigenfunctions with $\epsilon \rightarrow 0$, i.e., the long-wavelength modes with $q \rightarrow 0$, are essentially the same as in the uniform lattice and are therefore not localized.

\section{FLUCTUATION EXPANSION FOR RBM AND RJM}

Our method is a generalization of Zwanzig's calculation $^{12}$ for the response function $\mathscr{F}(q, z)$ in the RBM, which leads to a systematic expansion in powers of $\sqrt{z}$ as $z \rightarrow 0$ with $q=\kappa \sqrt{z}$ and $\kappa$ fixed. The essential point in this calculation is the proper choice of fluctuations: fluctuations $1 / w_{n}-\langle 1 / w\rangle$ around the exact inverse diffusion coefficient $D^{-1}=\langle 1 / w\rangle$. The method can be applied equally well to the isotropic RJM even for general dimensionality.

We start with the RBM, where the average Green's function (2.21) and the response function (2.20) are identical as explained below (2.21):

$$
\mathscr{F}(q, z)=\mathscr{G}(q, z)=\left\langle\left(z+f^{*} W f\right)^{-1}\right\rangle_{q q},
$$

where we have used (2.13). For $z \rightarrow 0$ the above functions reduce to

$$
\begin{aligned}
\mathscr{F}(q, 0) & =[f(q)]^{-1}\left\langle W^{-1}\right\rangle_{q q}\left[f^{*}(q)\right]^{-1} \\
& =[2 D(1-\cos q)]^{-1} .
\end{aligned}
$$

We want to expand the resolvent in the proper fluctuations around their value at $z=0$,

$$
\Delta=D W^{-1}-1
$$

or

$$
\Delta_{q q^{\prime}}=N^{-1} \sum_{n} \delta_{n} e^{i n\left(q-q^{\prime}\right)}
$$

with

$$
\delta_{n}=D\left(1 / w_{n}-\langle 1 / w\rangle\right),
$$

where $W^{-1}$ is the matrix inverse of $W$ as can be verified from (2.17) and (2.3). Using a matrix identity we write

$$
\begin{aligned}
\widehat{\Gamma}(z) & =\left(z+f^{*} W f\right)^{-1}=f^{-1} W^{-1}\left(z W^{-1}+f f^{*}\right)^{-1} f \\
& =f^{-1}(1+\Delta)(z+z \Delta+\omega)^{-1} f
\end{aligned}
$$

where we have used (2.17) to write

$$
\omega(q)=D f^{*}(q) f(q)=2 D(1-\cos q) .
$$

The function for the RBM follows from these equations as

$$
\mathscr{F}(q, z)=\left\langle(1+\Delta)(z+\omega+z \Delta)^{-1}\right\rangle_{q q} .
$$

If we neglect the fluctuations in (3.5) we find the Green's function $g_{0}(q, z)$ for a uniform lattice with a constant transition rate $D=1 /\left\langle w^{-1}\right\rangle$ :

$$
g_{0}(q, z)=[z+\omega(q)]^{-1} \text {. }
$$

In the following we use the abbreviated notation $g_{0}(q)$ for $g_{0}(q, z)$. In our systematic approach we expand the response function in powers of $\Delta$, which yields for the RBM,

$$
\mathscr{F}(q, z)=g_{0}(q)-z \omega(q) g_{0}^{2}(q) A(q, z)
$$

with

$$
g_{0}(q) A(q, z)=\sum_{l=0}^{\infty}(-z)^{l}\left\langle\left(\Delta g_{0}\right)^{l+2}\right\rangle_{q q}
$$

and

$$
\begin{array}{r}
\left\langle\left(\Delta g_{0}\right)^{l+1}\right\rangle_{q q}=\sum_{q_{1}, \ldots, q_{l}}\left\langle\Delta_{q q_{1}} \Delta_{q_{1} q_{2}} \cdots \Delta_{q_{l} q}\right\rangle \\
\times g_{0}\left(q_{1}\right) \cdots g_{0}\left(q_{l}\right) g_{0}(q) .
\end{array}
$$

Similarly we obtain the fluctuation expansion of the gen- 
eralized diffusion coefficient $U(q, z)$ defined in (2.4) with

$$
q^{2} U(q, z)=\omega(q)+\Gamma(q, z)
$$

and

$$
\begin{aligned}
\Gamma(q, z) & =[\mathscr{F}(q, z)]^{-1}-\left[g_{0}(q, z)\right]^{-1} \\
& =z \omega(q) A(q, z)\left[1-z \omega(q) g_{0}(q) A(q, z)\right]^{-1} .
\end{aligned}
$$

For the RJM the response function (2.20) contains the stationary initial distribution (1.5), $\psi_{n}=D / w_{n}$ through the matrix $\Psi$. It satisfies $\Psi=1+\Delta$ on account of (3.2a). Combination of (2.20) and (2.16) gives the response function for the RJM in the form

$$
\begin{aligned}
\mathscr{F}(q, z) & =\left\langle\left(z+f^{*} f W\right)^{-1}(1+\Delta)\right\rangle_{q q} \\
& =\left\langle(1+\Delta)(z+z \Delta+\omega)^{-1}(1+\Delta)\right\rangle_{q q} .
\end{aligned}
$$

Note that the average Green's function (2.21) for the RJM

$$
\begin{aligned}
\mathscr{G}(q, z) & =\left\langle\left(z+f^{*} f W\right)^{-1}\right\rangle_{q q} \\
& =\left\langle(1+\Delta)(z+z \Delta+\omega)^{-1}\right\rangle_{q q}
\end{aligned}
$$

is identical to the response function (3.5) in the RBM. The response function $\mathscr{F}(q, z)$ and the generalized diffusion coefficient $U(q, z)$ or $\Gamma(q, z)$ defined in (3.10) can again be expanded in powers of $\Delta$ :

$$
\begin{aligned}
& \mathscr{F}(q, z)=g_{0}(q)+\omega^{2}(q) g_{0}^{2}(q) A(q, z) \\
& \Gamma(q, z)=-\omega^{2}(q) A(q, z)\left[1+\omega^{2}(q) g_{0}(q) A(q, z)\right]^{-1}
\end{aligned}
$$

where $A(q, z)$ is given in (3.8).

In order to calculate the terms in the fluctuation expansion of $A(q, z)$ we have developed a diagrammatic expansion. As the method is rather involved it is presented in a separate appendix, Appendix A. The result of these calculations, with sixth-order fluctuations included [i.e., terms with $l=0, \ldots, 4$ included in the definition (3.8) of $A(q, z)$ ], is given by the following formula, which is applied to one-dimensional hopping models in Sec. IV and to higher-dimensional ones in Sec. V:

$$
\begin{aligned}
A(q, z)= & \kappa_{2} h_{1}-z \kappa_{3} h_{1}^{2}+z^{2}\left[\kappa_{4} h_{1}^{3}+\kappa_{2}^{2}\left(g_{0} h_{1}^{2}+g_{1}+h_{1} h_{2}\right)\right]-z^{3} \kappa_{2} \kappa_{3}\left(2 g_{0} h_{1}^{3}+3 h_{1}^{2} h_{2}+4 g_{1} h_{1}+\tilde{h}_{1}\right) \\
& +z^{4} \kappa_{2}^{3}\left(g_{0}^{2} h_{1}^{3}+2 g_{0} h_{1} g_{1}+2 g_{0} h_{1}^{2} h_{2}+h_{1}^{2} h_{3}+h_{1} h_{2}^{2}+\widetilde{h}_{2}+3 g_{2} h_{1}+4 g_{3}\right)+\cdots
\end{aligned}
$$

The functions $g_{n}(n=1,2,3, \ldots)$ defined in (B5) of Appendix B depend on $q$ and $z$ whereas $h_{n}(z)$ and $\tilde{h}_{n}(z)$, defined in (B1) and (B9), depend only on $z$. The cumulants $\kappa_{l}=\left\langle\left\langle\delta^{l}\right\rangle\right\rangle$ are given in terms of moments $\mu_{l}=\left\langle\delta^{l}\right\rangle$ with $\delta=D\left(w^{-1}-\left\langle w^{-1}\right\rangle\right)=D / w-1$. The first few of them are given by

$$
\begin{aligned}
& \kappa_{2}=\mu_{2}, \quad \kappa_{3}=\mu_{3}, \\
& \kappa_{4}=\mu_{4}-3 \mu_{2}^{2}, \quad \kappa_{5}=\mu_{5}-10 \mu_{3} \mu_{2}, \\
& \kappa_{6}=\mu_{6}-15 \mu_{4} \mu_{2}-10 \mu_{3}^{2}+30 \mu_{2}^{3} .
\end{aligned}
$$

We have assumed that the $\mu_{l}$ with $l \leq 6$ exist.

The main goal of this paper is to present a systematic calculation of the small- $z$ and $-q$ behavior of the response function, in particular for the range of $q$ values with $q$ of order $\kappa \sqrt{z}$ (with $\kappa$ fixed) or smaller. For the onedimensional case we have made a systematic analysis of the small- $q$ and $-z$ behavior of all terms in the fluctuation expansion, which is presented in Appendix B. There it is shown that $\left\langle\left(z \Delta g_{0}\right)^{l}\right\rangle_{q q}$ is of order $z^{\alpha(l)}$ with $\alpha(l)=\frac{1}{2}\left[\frac{1}{2}(l+1)\right]$ to leading order for small $z$ with $q \simeq \kappa \sqrt{z}$. The function $[x]$ is the largest integer smaller than or equal to $x$. Hence the perturbation expansion (3.15) starts with a term of order $z^{-1 / 2}$ in the onedimensional case and is exact to order $z^{1 / 2}$ terms included. The contributions to (3.15) from $\left\langle\left(\Delta g_{0}\right)^{6}\right\rangle$, proportional to $\kappa_{3}^{2}, \kappa_{4} \kappa_{2}$, and $\kappa_{6}$, should be neglected as they are at least of order $z$ [see (B14)].

\section{RESULTS FOR ONE-DIMENSIONAL HOPPING MODELS}

From the results of the preceding section and of Appendixes $\mathbf{A}$ and $\mathbf{B}$ one can calculate the frequency-dependent diffusion coefficient $U_{0}(z)$ and modified Burnett coefficient $U_{2}(z)$, defined in (2.7), at low frequencies, as well as the long-time behavior of the moments of displacement. We further calculate the long-time behavior of the staying probability $P_{0}(t)$ and the small-z behavior of the singlesite Green's function $\hat{G}_{0}(z)$, which describes the density of eigenstates $\mathscr{N}(\epsilon)$ and the inverse localization length $\gamma(\epsilon)$ at the lower end of the eigenvalue spectrum.

To calculate $U_{0}(z)$ and $U_{2}(z)$ we need the $q$ expansion (2.7) of $q^{2} U(q, z)=\omega(q)+\Gamma(q, z)$. As $\Gamma(q, z)$ has been expressed in (3.11) and (3.14) in terms of $A(q, z)$, we also introduce its $q$ expansion:

$$
A(q, z)=A_{0}(z)-q^{2} A_{2}(z)+\cdots .
$$

By collecting the results of Appendix B to the relevant order in $z$ one finds from (3.15)

$$
\begin{aligned}
& D A_{0}(z)=\frac{1}{2} \kappa_{2}(D / z)^{1 / 2}+a_{0}+a_{1}(z / D)^{1 / 2}+O(z), \\
& D A_{2}(z)=b_{0} D / z+b_{1}(D / z)^{1 / 2}+O\left(z^{0}\right),
\end{aligned}
$$

where the coefficients $a_{l}$ and $b_{l}$, listed in Table I, have been expressed in the cumulants $\kappa_{l}$. Coefficients with $l=0$ involve at most fourth-order fluctuations, those with $l=1$ sixth-order fluctuations.

For the RBM the coefficients $U_{0}(z)$ and $U_{2}(z)$, occurring in the $q$ expansion (2.7) of the generalized diffusion coefficient, can be expressed in $A_{0}$ and $A_{2}$ with the help of (3.10) and (3.11):

$$
\begin{aligned}
& U_{0}(z)=D+z D A_{0}(z), \\
& U_{2}(z)=\frac{1}{12} D+z\left[D A_{2}(z)+\frac{1}{12} D A_{0}(z)-D^{2} A_{0}^{2}(z)\right],
\end{aligned}
$$

where also the $q$ expansion of $\omega(q)$ in (3.4) has been used. Substitution of (4.2) in (4.3) gives 
TABLE I. Coefficients in terms of cumulants.

\begin{tabular}{lll}
\hline \hline & \multicolumn{1}{c}{ Exact } & \multicolumn{1}{c}{ EMA } \\
\hline$a_{0}$ & $-\frac{1}{4} \kappa_{3}+\frac{11}{24} \kappa_{2}^{2}$ & $-\frac{1}{4} \kappa_{3}+\frac{3}{8} \kappa_{2}^{2}$ \\
$a_{1}$ & $\frac{1}{8} \kappa-\frac{1}{16} \kappa_{2}-\frac{61}{96} \kappa_{2} \kappa_{3}+\frac{1345}{2304} \kappa_{2}^{3}$ & $\frac{1}{8} \kappa_{4}-\frac{1}{16} \kappa_{2}-\frac{7}{16} \kappa_{2} \kappa_{3}+\frac{21}{64} \kappa_{2}^{3}$ \\
$b_{0}$ & $\frac{7}{27} \kappa_{2}^{2}$ & $\frac{1}{4} \kappa_{2}^{2}$ \\
$b_{1}$ & $-\frac{29}{108} \kappa_{2} \kappa_{3}+\frac{107}{216} \kappa_{2}^{3}$ & $-\frac{1}{4} \kappa_{2} \kappa_{3}+\frac{3}{8} \kappa_{2}^{3}$ \\
$c_{0}$ & $\frac{1}{12}+\frac{1}{108} \kappa_{2}^{2}$ & $\frac{1}{12}$ \\
$c_{1}$ & $\frac{1}{24} \kappa_{2}-\frac{1}{54} \kappa_{2} \kappa_{3}+\frac{1}{27} \kappa_{2}^{3}$ & $\frac{1}{24} \kappa_{2}$ \\
$d_{0}$ & $\frac{1}{12}-\frac{1}{2} \kappa_{3}+\frac{127}{108} \kappa_{2}^{2}$ & $\frac{1}{12}-\frac{1}{2} \kappa_{3}+\kappa_{2}^{2}$ \\
$d_{1}$ & $-\frac{1}{12} \kappa_{2}+\frac{1}{4} \kappa_{4}-\frac{665}{432} \kappa_{2} \kappa_{3}+\frac{5747}{3456} \kappa_{2}^{3}$ & $-\frac{1}{12} \kappa_{2}+\frac{1}{4} \kappa_{4}-\frac{9}{8} \kappa_{2} \kappa_{3}+\frac{33}{32} \kappa_{2}^{3}$ \\
$r_{0}$ & $\frac{1}{16}\left(-1+\kappa_{3}-\frac{15}{16} \kappa_{2}^{2}\right)$ & $\frac{1}{16}\left(-1+\kappa_{3}-\frac{3}{4} \kappa_{2}^{2}\right)$ \\
$r_{1}$ & $\frac{1}{16}\left(\kappa_{2}-\frac{1}{2} \kappa_{4}+\frac{3}{2} \kappa_{2} \kappa_{3}-\frac{15}{16} \kappa_{2}^{3}\right)$ & $\frac{1}{16}\left(\kappa_{2}-\frac{1}{2} \kappa_{4}+\kappa_{2} \kappa_{3}-\frac{1}{2} \kappa_{2}^{3}\right)$ \\
$s_{0}$ & $-\frac{1}{4} \kappa_{2}-\frac{1}{4} \kappa_{3}+\frac{1}{8} \kappa_{2}^{2}$ & exact \\
$s_{1}$ & $\frac{1}{16}\left(-\kappa_{2}+2 \kappa_{3}+2 \kappa_{4}+\frac{3}{2} \kappa_{2}^{2}-\frac{7}{2} \kappa_{2} \kappa_{3}+\frac{25}{16} \kappa_{2}^{3}\right)$ & $\frac{1}{16}\left(-\kappa_{2}+2 \kappa_{3}+2 \kappa_{4}-3 \kappa_{2} \kappa_{3}+\frac{5}{4} \kappa_{2}^{3}\right)$ \\
\hline \hline
\end{tabular}

$$
\begin{gathered}
U_{0}(z)=D\left[1+\frac{1}{2} \kappa_{2}(z / D)^{1 / 2}+a_{0}(z / D)\right. \\
\left.+a_{1}(z / D)^{3 / 2}+O\left(z^{2}\right)\right], \\
U_{2}(z)=D\left[c_{0}+c_{1}(z / D)^{1 / 2}+O(z)\right],
\end{gathered}
$$

with

$$
\begin{aligned}
& c_{0}=\frac{1}{12}+b_{0}-\frac{1}{4} \kappa_{2}^{2}, \\
& c_{1}=b_{1}-a_{0} \kappa_{2}+\frac{1}{24} \kappa_{2} .
\end{aligned}
$$

Expressions for $c_{0}$ and $c_{1}$ in terms of cumulants $\kappa_{l}$ are given in Table $\mathbf{I}$.

For the RJM we obtain similarly from (3.10) and (3.14)

$$
\begin{aligned}
U_{0}(z) & =D \\
U_{2}(z) & =\frac{1}{12} D+D^{2} A_{0}(z) \\
& =D\left[\frac{1}{2} \kappa_{2}(D / z)^{1 / 2}+\frac{1}{12}+a_{0}+a_{1}(z / D)^{1 / 2}+O(z)\right] .
\end{aligned}
$$

In the last line of (4.6) we have used (4.2). The most striking difference between (4.4) and (4.6) regarding $U_{0}(z)$ is the occurrence of a $\sqrt{z}$ singularity in the RBM and the absence of any singular terms in the RJM, and regarding $U_{2}(z)$ the weaker $\sqrt{z}$ singularity in the RBM and the stronger $1 / \sqrt{z}$ singularity in the RJM.

Next, we consider the moments of displacement $\left\langle n^{l}\right\rangle_{t}$, which have been expressed in $U_{0}$ and $U_{2}$ in (2.8). For the RBM the long-time behavior of the mean-square displacement follows from (2.8) and (4.4) as

$$
\begin{aligned}
\frac{1}{2}\left\langle n^{2}\right\rangle_{t}= & D t+\kappa_{2}(D t / \pi)^{1 / 2}+a_{0}+a_{1}(\pi D t)^{-1 / 2} \\
& +O\left(t^{-3 / 2}\right) .
\end{aligned}
$$

To dominant order the average mean-square displacement is described in terms of an effective diffusion coefficient $D=1 /\left\langle w^{-1}\right\rangle$ for a corresponding uniform lattice, around which we expand in fluctuations. The second term represents a long-time tail, which is absent in random walks on uniform lattices, since $\kappa_{2}=D^{2}\left\langle\left(w^{-1}\right.\right.$ $\left.\left.-\left\langle w^{-1}\right\rangle\right)^{2}\right\rangle$ vanishes. Also the higher corrections, $a_{0}$ and $a_{1}$, are vanishing on a uniform lattice. The long-time behavior of the VACF $\varphi_{2}(t)$ is given by the second derivative of (4.7). It has the well-known long-time tail $t^{-(d+2) / 2}$ ( $d$ is the dimensionality), typical of diffusion in stationary random media, such as the Lorentz gas. ${ }^{15,21}$

The long-time behavior of the fourth moment follows similarly from (2.8), (4.4) and (4.5):

$$
\begin{aligned}
\frac{1}{4 !}\left\langle n^{4}\right\rangle_{t}= & \frac{1}{2} D^{2} t^{2}+(4 / 3 \sqrt{\pi}) \kappa_{2}(D t)^{3 / 2} \\
& +d_{0} D t+2 d_{1}(D t / \pi)^{1 / 2}+O\left(t^{0}\right)
\end{aligned}
$$

with the coefficients $d_{l}$ defined as

$$
\begin{aligned}
& d_{0}=c_{0}+2 a_{0}+\frac{1}{4} \kappa_{2}^{2}, \\
& d_{1}=c_{1}+2 a_{1}+a_{0} \kappa_{2},
\end{aligned}
$$

and listed in Table I.

The above results are also sufficient to calculate the fourth cumulant $\left\langle\left\langle n^{4}\right\rangle\right\rangle_{t}=\left\langle n^{4}\right\rangle_{t}-3\left(\left\langle n^{2}\right\rangle_{t}\right)^{2}$, where the leading term behaves as $\kappa_{2} t^{+3 / 2}$ for $t \rightarrow \infty$. The long-time behavior of $(d / d t)\left\langle\left\langle n^{4}\right\rangle\right\rangle_{t}=B(t)$ increases proportional to $\kappa_{2} \sqrt{t}$ as $t \rightarrow \infty$. In a uniform lattice this term vanishes and $B(t)$ approaches a finite limit $B(\infty)=D / 12$, which is called the super-Burnett transport coefficient.

One sometimes considers ${ }^{19,27}$ a modified Burnett coefficient $U_{2}(0)$, which is, according to (4.4), given by $U_{2}(0)=c_{0} D$ in the RBM. The Burnett correlation function $\varphi_{4}(t)$, which is the inverse Laplace transform of $U_{2}(z)$, has a long-time tail $\varphi_{4} \sim t^{-3 / 2}$ with a coefficient that vanishes on a uniform lattice.

In the RJM the mean-square displacement, deduced from (2.8) and (4.6), satisfies the remarkably simple form $\left\langle n^{2}\right\rangle_{t}=2 D t$, valid for all times, as was first shown by Haus et al. ${ }^{7}$ The VACF for this model $\varphi_{2}(t)=D \delta_{+}(t)$ has 
no long-time tail. However, long-time effects caused by the randomness in the medium, occur in $\left\langle n^{4}\right\rangle_{t}$. Its longtime behavior can be deduced from (2.8) and (4.6) and is given by

$$
\begin{aligned}
\frac{1}{4 !}\left\langle n^{4}\right\rangle_{t}= & \frac{1}{2} D^{2} t^{2}+\frac{2}{3 \sqrt{\pi}} \kappa_{2}(D t)^{3 / 2} \\
& +\left(\frac{1}{12}+a_{0}\right) D t+2 a_{1}(D t / \pi)^{1 / 2}+O\left(t^{0}\right) .
\end{aligned}
$$

The cumulant $\left\langle\left\langle n^{4}\right\rangle\right\rangle$ is again proportional to $\kappa_{2} t^{3 / 2}$, which represents a long-time tail, vanishing on a uniform lattice. In the RJM the quantities $B(\infty)$ and $U_{2}(0)$ are divergent due to the effects of long-time tails and the Burnett correlation function $\varphi_{4}(t)$ has a long-time tail $\sim t^{-1 / 2}$, to be compared with $\varphi_{4}(t) \sim t^{-3 / 2}$ in the RBM.

The probabilities of displacement $P_{n}(t)$ can also be obtained from the small-z, $-q$ behavior of the response function $\mathscr{F}(q, z)$ through Fourier inversion of (2.2). We are specially interested in the probability of zero displacement or staying probability $P_{0}(t)$ in case of a stationary initial distribution [see (2.10) and (2.11)]. Its Laplace transform is given by

$$
\hat{P}_{0}(z)=N^{-1} \sum_{q} \mathscr{F}(q, z)
$$

Furthermore we are interested in the average single-site Green's function $\hat{G}_{0}(z)$, corresponding to the staying probability in case of a uniform initial distribution [see (2.23)]:

$$
\hat{G}_{0}(z)=N^{-1} \sum_{q} \mathscr{G}(q, z) .
$$

The latter function determines the spectral properties of the eigenmodes of the system [see Eqs. (2.22)-(2.26)]. In the RBM both functions coincide; in the RJM they do not. Starting with the RBM, where $\mathscr{F}=\mathscr{G}$ and $\hat{P}_{0}=\widehat{G}_{0}$ according to (3.1), it follows from (3.7) and (B1) in Appen$\operatorname{dix} B$ that

$$
\hat{G}_{0}(z)=h_{1}(z)-z N^{-1} \sum_{q} \omega(q) g_{0}^{2}(q) A(q, z) .
$$

By substituting (3.15) for $A(q, z)$ and introducing the functions $k_{n}(z), f_{n}(z)$, and $\widetilde{f}_{n}(z)$, defined in (B4) and (B11) of Appendix B, we find by including fluctuations up to sixth order

$$
\begin{aligned}
\hat{G}_{0}(z)= & h_{1}-z \kappa_{2} h_{1} h_{2}+z^{2} \kappa_{3} h_{1}^{2} h_{2}-z^{3}\left[\kappa_{4} h_{1}^{3} k_{2}+\kappa_{2}^{2}\left(h_{1}^{2} k_{3}+f_{1}+h_{1} h_{2} k_{2}\right)\right]+z^{4} \kappa_{2} \kappa_{3}\left(2 h_{1}^{3} k_{3}+3 h_{1}^{2} h_{2} k_{2}+4 h_{1} f_{1}+\tilde{h}_{1} k_{2}\right) \\
& -z^{5} \kappa_{2}^{3}\left[h_{1}^{3} k_{4}+2 h_{1} \widetilde{f}_{1}+2 h_{1}^{2} h_{2} k_{3}+\left(h_{1}^{2} h_{3}+h_{1} h_{2}^{2}+\tilde{h}_{2}\right) k_{2}+3 h_{1} f_{2}+4 f_{3}\right]+\cdots
\end{aligned}
$$

This function is given in full detail as it is also needed in Sec. V for higher-dimensional systems. From the small-z behavior of these functions as obtained in Appendix B we find

$$
\begin{aligned}
\hat{G}_{0}(z)=D^{-1}[ & \frac{1}{2}(D / z)^{1 / 2}-\frac{1}{8} \kappa_{2}+r_{0}(z / D)^{1 / 2} \\
& \left.+r_{1}(z / D)+O\left(z^{3 / 2}\right)\right]
\end{aligned}
$$

where the coefficients $r_{0}$ and $r_{1}$ are listed in Table I. This result is in complete agreement with the low-frequency behavior of the characteristic function $\Omega(z)$ in a harmonic chain with random masses as determined by Nieuwenhuizen, ${ }^{24}$ where $\hat{G}_{0}(z)=d \Omega(z) / d z$. The $\kappa_{l}$ in this case are cumulants of the mass distribution. In this paper the small-z behavior is determined explicitly up to terms of order $z^{7 / 2}$ included. It is used to calculate the specific heat for harmonic chains with random masses. The longtime behavior of the staying probability also follows from (4.14) with $\hat{G}_{0}(z)=\hat{P}_{0}(z)$ in the $\mathrm{RBM}$ :

$$
P_{0}(t) \simeq(4 \pi D t)^{-1 / 2}-\left(r_{0} / 2 \sqrt{\pi}\right)(D t)^{-3 / 2}+O\left(t^{-5 / 2}\right) .
$$

Furthermore, the single-site Green's function $\hat{G}_{0}(z)$ determines the density of eigenstates $\mathscr{N}(\epsilon)$ through (2.22) and the inverse localization length $\gamma(\epsilon)$ through (2.26). Since $\widehat{G}_{0}(z)$ is known through (4.15) for small $z$, we have for the lower end of the spectrum $(\epsilon \rightarrow 0)$ :

$$
\mathscr{N}(\epsilon)=(2 \pi)^{-1}(D \epsilon)^{-1 / 2}-\left(r_{0} / \pi\right) D^{-3 / 2} \epsilon^{1 / 2}+O\left(\epsilon^{3 / 2}\right)
$$

and

$$
\gamma(\epsilon)=\frac{1}{8} \kappa_{2} \epsilon / D+\frac{1}{2} r_{1}(\epsilon / D)^{2}+O\left(\epsilon^{3}\right)
$$

Using the replica method ${ }^{9}$ Stephen and Kariotis obtained the same result for the density of states. For the inverse localization length they only obtained the first term on the right-hand side of (4.17). The Green's function $\hat{G}_{0}(z)$ for the RJM is identical to the one in the RBM by virtue of (3.13) and so are the spectral properties. We restrict ourselves to the staying probability $P_{0}(t)$, which can be obtained from (4.11) and (3.14) in the form

$$
\begin{aligned}
\hat{P}_{0}(z) & =h_{1}(z)+N^{-1} \sum_{q} \omega^{2}(q) g_{0}^{2}(q) A(q, z) \\
& =\hat{G}_{0}(z)+\hat{E}(z)
\end{aligned}
$$

where $\hat{G}_{0}(z)$ is the single-site Green's function (4.14), which also applies to the RJM and where

$$
\hat{E}(z)=N^{-1} \sum_{q} \omega(q) g_{0}(q) A(q, z)
$$

In deriving (4.18) we used the relation $\omega(q) g_{0}(q)$ $=1-z g_{0}(q)$. By inserting the fluctuation expansion (3.15) into (4.19) and by introducing the functions $\bar{f}_{n}(z)$ defined in (B11) of Appendix B we obtain 


$$
\begin{aligned}
\widehat{E}(z)= & \kappa_{2} h_{1} k_{1}-z \kappa_{3} h_{1}^{2} k_{1}+z^{2}\left[\kappa_{4} h_{1}^{3} k_{1}+\kappa_{2}^{2}\left(h_{1}^{2} k_{2}+\bar{f}_{1}+h_{1} h_{2} k_{1}\right)\right] \\
& -z^{3} \kappa_{2} \kappa_{3}\left(3 h_{1}^{2} h_{2}+\widetilde{h}_{1}\right) k_{1}+z^{4} \kappa_{2}^{3}\left(h_{1}^{2} h_{3}+h_{1} h_{2}^{2}+\widetilde{h}_{2}\right) k_{1}+\cdots .
\end{aligned}
$$

With the help of (B12) it results in the following small-z behavior:

$$
\widehat{E}(z)=D^{-1}\left[\frac{1}{2} \kappa_{2}(D / z)^{1 / 2}+s_{0}+s_{1}(z / D)^{1 / 2}+O(z)\right],
$$

where the coefficients $s_{0}$ and $s_{1}$ are listed in Table I. The long-time behavior of the staying probability follows by adding (4.21) and (4.15) and yields after Laplace inversion

$$
\begin{aligned}
P_{0}(t) \simeq & \left(1+\kappa_{2}\right)(4 \pi D t)^{-1 / 2} \\
& -\left[\left(s_{1}+r_{0}\right) / 2 \sqrt{\pi}\right](D t)^{-3 / 2}+O\left(t^{-5 / 2}\right) .
\end{aligned}
$$

It is of interest to compare the properties of both hopping models. The response function $\mathscr{F}(q, z)$ to lowest order in $z$ (with $q=\kappa \sqrt{z}$ and $\kappa$ fixed) is the same as in the uniform chain with an effective diffusion coefficient $D=1 /\left\langle w^{-1}\right\rangle$. The same holds for the dominant, longtime behavior of $\left\langle n^{2}\right\rangle_{t}$ and $\left\langle n^{4}\right\rangle_{t}$ [see $\left.(4.7)-(4.10)\right]$. Here, the effects of randomness show up in correction terms of order $\sqrt{z}$ or $1 / \sqrt{t}$. The same dominant behavior can again be seen in the staying probability $P_{0}(t)$ [see (4.16)] of the RBM. However, in the RJM the fluctuations in the random medium increase the dominant, long-time behavior of $P_{0}(t)$ in (4.22) by a factor $\left(1+\kappa_{2}\right)$, when compared to the staying probability on a uniform chain with effective diffusion coefficient $D=1 /\left\langle w^{-1}\right\rangle$.

\section{RJM IN $d$ DIMENSIONS}

The results of the previous sections can be trivially generalized to $d$-dimensional systems for the isotropic RJM. Consider a hypercubic lattice with $N^{d}$ sites, labeled $\overrightarrow{\mathrm{n}}$, with unit lattice distance and periodic boundary conditions. A random jump rate $v_{\vec{n}}$ is assigned to the site $\vec{n}$, from where the hopping particle jumps to a nearestneighbor site with a transition rate $w_{\overrightarrow{\mathrm{n}}}=v_{\overrightarrow{\mathrm{n}}} / 2 d$. The master equation for this model reads

$$
\dot{p}_{\overrightarrow{\mathrm{n}}}=-\sum_{\alpha=1}^{d}\left(2-E_{\overrightarrow{\mathrm{n}} \alpha}-E_{\overrightarrow{\mathrm{n}} \alpha}^{-1}\right) w_{\overrightarrow{\mathrm{n}}} p_{\overrightarrow{\mathrm{n}}},
$$

where $E_{\overrightarrow{\mathrm{n}} \alpha} \overrightarrow{\mathrm{n}}=\overrightarrow{\mathrm{n}}+\overrightarrow{\mathrm{e}}_{\alpha}$ with $\overrightarrow{\mathrm{e}}_{\alpha}$ a unit vector in the $\alpha$ direction. Haus et al. ${ }^{7}$ have shown that the relation $\left\langle n_{\alpha}^{2}\right\rangle_{t}=2 D t$ with $D^{-1}=\left\langle w^{-1}\right\rangle$ is valid for all times if one starts with the stationary initial distribution. This implies the absence of long-time tails in the VACF $\varphi_{2}(t)$. Nevertheless long-time tails do occur in the fourth moment, as we will see below. After introduction of Fourier transforms most steps of Secs. II and III can be reiterated and one obtains for the response function [cf. (3.12)]

$$
\begin{aligned}
\mathscr{F}(\overrightarrow{\mathrm{q}}, z) & =\left\langle(z+\omega W / D)^{-1}(1+\Delta)\right\rangle_{\overrightarrow{\mathrm{q}} \overrightarrow{\mathrm{q}}} \\
& =\left\langle(1+\Delta)(z+\omega+z \Delta)^{-1}(1+\Delta)\right\rangle_{\overrightarrow{\mathrm{q}} \overrightarrow{\mathrm{q}}},
\end{aligned}
$$

where

$$
\begin{aligned}
\omega(\overrightarrow{\mathrm{q}}) & =D \sum_{\alpha=1}^{d} f^{*}\left(q_{\alpha}\right) f\left(q_{\alpha}\right) \\
& =2 D \sum_{\alpha=1}^{d}\left(1-\cos q_{\alpha}\right)
\end{aligned}
$$

and

$$
\Delta_{\overrightarrow{\mathrm{q}} \overrightarrow{\mathrm{q}}}=D N^{-d} \sum_{\overrightarrow{\mathrm{n}}}\left[1 / w_{\overrightarrow{\mathrm{n}}}-\left\langle\frac{1}{w}\right\rangle\right] \exp \left[i \overrightarrow{\mathrm{n}} \cdot\left(\overrightarrow{\mathrm{q}}-\overrightarrow{\mathrm{q}}^{\prime}\right)\right] .
$$

Its fluctuation expansion is given by

$$
\mathscr{F}(\overrightarrow{\mathrm{q}}, z)=g_{0}(\overrightarrow{\mathrm{q}})+\omega^{2}(\overrightarrow{\mathrm{q}}) g_{0}^{2}(\overrightarrow{\mathrm{q}}) A(\overrightarrow{\mathrm{q}}, z),
$$

with $A(\overrightarrow{\mathrm{q}}, z)$ defined by (3.8) and calculated up to sixthorder fluctuation contributions included in (3.15). The lattice functions $h_{n}^{(d)}(z), g_{n}^{(d)}(\overrightarrow{\mathrm{q}}, z)$, etc., are the $d$ dimensional analogs of the corresponding one-dimensional expressions $h_{n}(z), g_{n}(\overrightarrow{\mathrm{q}}, z)$, etc. The same applies to the Laplace transform of the staying probability, $\hat{P}_{0}(z)$, given in (4.18)-(4.20). The problem is therefore reduced to an analysis of the $d$-dimensional lattice functions. Here we restrict ourselves to the contributions from second-order fluctuations and obtain by virtue of (3.15) and (4.1)

$$
A(\overrightarrow{\mathrm{q}}, z) \simeq A_{0}(z) \simeq \kappa_{2} h_{1}^{(d)}(z),
$$

with

$h_{1}^{(d)}(z)=(2 \pi)^{-d} \int_{-\pi}^{\pi} \cdots \int_{-\pi}^{\pi} d q_{1} \cdots d q_{d}[z+\omega(\overrightarrow{\mathrm{q}})]^{-1}$.

The function $h_{1}^{(d)}(z)$ is the single-site Green's function for a uniform $d$-dimensional hypercubic lattice with diffusion constant $D=\langle 1 / w\rangle^{-1}$. For two-dimensional square lattices it yields ${ }^{32}$

$$
h_{1}^{(2)}(z)=\frac{2}{\pi(z+4 D)} K\left(\frac{4 D}{z+4 D}\right),
$$

where $K(u)$ is the complete elliptic integral of the first kind. ${ }^{33}$ Its dominant small- $z$ behavior is

$$
h_{1}^{(2)}(z) \simeq-(1 / 8 \pi D) \ln (z / 4 D) \text {. }
$$

For a three-dimensional simple cubic lattice $h_{1}^{(3)}(z)$ is an integral over an elliptic function $K(u)$ (Ref. 34) and its small-z behavior is $h_{1}^{(3)}(z) \simeq h_{1}^{(3)}(0)-\left(4 \pi D^{3 / 2}\right)^{-1} \sqrt{z}$ $+\cdots$. The long-time tail in the fourth moment follows from (4.6) and (2.8) as

$$
\left(\frac{d}{d t}\right)^{2}\left\langle n_{x}^{4}\right\rangle_{t} \simeq 4 ! D^{2}\left[1+\kappa_{2} H^{(d)}(t)\right],
$$

where $H(t)$ is the inverse Laplace transform of $h_{1}(z)$ (Ref. 1) 


$$
\begin{aligned}
H^{(d)}(t) & =\left[H^{(1)}(t)\right]^{d}=e^{-2 d D t}\left[I_{0}(2 D t)\right]^{d} \\
& \simeq(4 \pi D t)^{-d / 2} \text { as } t \rightarrow \infty
\end{aligned}
$$

Here $I_{0}(x)$ is a modified Bessel function. For the longtime behavior of the staying probability we find an expression analogous to (4.22):

$$
P_{\overrightarrow{0}}(t) \simeq\left(1+\kappa_{2}\right)(4 \pi D t)^{-d / 2} .
$$

Analogous results can be derived for the low-frequency behavior of the single-site Green's function $\hat{G}_{\overrightarrow{0}}(z)$, which determines the spectral properties of relaxational modes in $d$-dimensional systems.

\section{DISCUSSION}

In order to study diffusion properties of onedimensional hopping models we have developed an expansion in powers of the fluctuation $\delta_{n}=\left(w_{n}^{-1}-\left\langle w^{-1}\right\rangle\right) /\left\langle w^{-1}\right\rangle$ around the exact diffusion coefficient $D=1 /\left\langle w^{-1}\right\rangle$, using a diagrammatic method. This has been done for two types of hopping models: the RBM, in which the random variables are assigned to the bonds, and the RJM, in which they are assigned to the sites. For small frequencies $(z \rightarrow 0)$ this fluctuation expansion is shown to yield a systematic expansion of the response function $\mathscr{F}(q, z)$, and the average Green's function $\mathscr{G}(q, z)$ in powers of $\sqrt{z}$ for small $z$ with $q \simeq \kappa \sqrt{z}$ and $\kappa$ kept fixed. This expansion does not cover the full $(q, z)$ dependence of $\mathscr{F}(q, z)$ for small $q$ and $z$. The region where $q$ tends to zero first is not covered by our results, as can be illustrated by calculating $U(0, z)$ and $U(q, 0)$, defined in (2.4), for the RJM. From (2.7) and (4.6) one sees that

$$
\lim _{z \rightarrow 0} U(0, z)=D
$$

Next we obtain from (3.12), (3.2), and (A3)

$$
\mathscr{F}(q, 0)=\left(1+\kappa_{2}\right) / \omega(q)
$$

so that

$$
\lim _{q \rightarrow 0} U(q, 0)=D /\left(1+\kappa_{2}\right) .
$$

The transport properties can be obtained from $\mathscr{F}(q, z)$ [see $(2.1)-(2.8)]$ and the spectral properties from $\mathscr{G}(q, z)$ [see (2.22)-(2.26)].

The difference between $\mathscr{F}$ and $\mathscr{G}$ is the following: In the definition $(2.20)$ of $\mathscr{F}(q, z)$ occurs an average over all starting positions of the hopping particle with a weight function $\psi_{n}$, that is the stationary solution of the master equation (1.1) for a frozen set of random variables $\left\{w_{n}\right\}$. This equilibrium weight $\psi_{n}$ may depend on the site label $n$, as is the case in the RJM. In the definition (2.21) of $\mathscr{G}(q, z)$ the possible starting positions of the hopping particle are given an equal weight.

The frequency-dependent transport coefficient $U_{0}(z)$ for the $R B M$ is given in (4.4). The leading contributions $U_{0}(z) \simeq D+\frac{1}{2} \kappa_{2}(D z)^{1 / 2}+\cdots$ have been obtained before by exact calculations, ${ }^{12}$ by the effective-medium approximation (EMA), 810 by renormalization-group methods, ${ }^{2}$ and by mode-coupling theories. ${ }^{20}$ The higher-order terms in (4.4) of order $z$ and of order $z^{3 / 2}$ are new results.

There exist approximate calculations based on the EMA by Webman and Klafter ${ }^{10}$ to order $z$ and by Haus et al. ${ }^{8}$ to order $z^{3 / 2}$, which differ already in terms of order $z$ from the exact results (4.4), contrary to the suggestion made in Refs. 8 and 10. For our comparison with the terms of order $z^{3 / 2}$ we have used the results of Ref. 8 , in which clusters containing three sites have been used instead of the lower approximation of two-site clusters. It appears that the approximate EMA results are completely accounted for by the contributions from diagrams in Fig. 1 , that are built as series-parallel circuits of the first diagram element (the so-called hypernetted-chain diagrams), their analytical contributions can be simply expressed in the contribution $h_{1}(z)$ of the first diagram (see Appendix A). The diagrams in Fig. 1, whose contribution contain $g_{n}(q, z)(n \geq 1)$ or $\widetilde{h}_{n}(z)(n \geq 1)$, do not have a series-parallel structure. The EMA is therefore the hypernetted-chain (HNC) approximation ${ }^{22,23}$ of our diagrammatic method. Table I gives the EMA values together with the exact ones of all coefficients appearing in Sec. IV.

For the VACF $\varphi_{2}(t)$ in the RBM, which is the inverse Laplace transform of $U_{0}(z)$ in (4.4), we also obtain

$$
\begin{aligned}
\varphi_{2}(t) \simeq & -\frac{1}{4} \kappa_{2}(D / \pi)^{1 / 2} t^{-3 / 2} \\
& +\frac{3}{4} a_{1}(\pi D)^{-1 / 2} t^{-5 / 2}+O\left(t^{-7 / 2}\right) .
\end{aligned}
$$

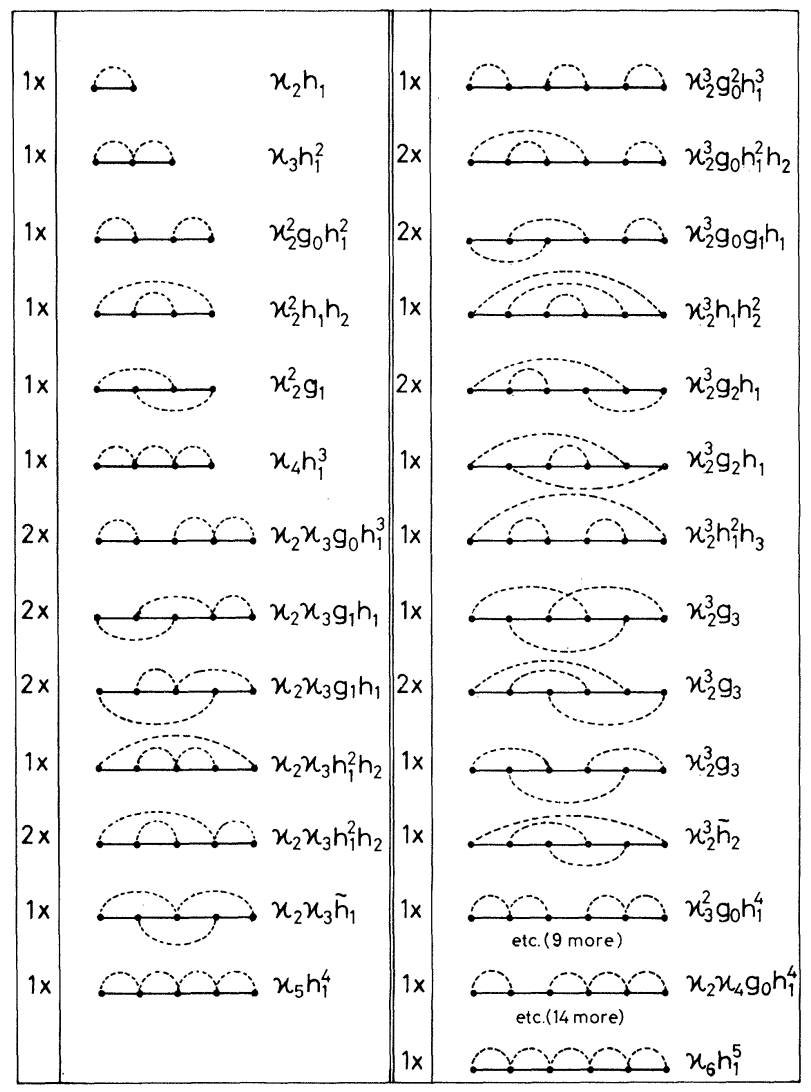

FIG. 1. Diagrams representing the terms $\left\langle\left(\Delta g_{0}\right)^{l} \Delta\right\rangle_{q q}$ in the fluctuation expansion $(l=1,2, \ldots, 5)$. 
The first term represents the well-known long-time tail $\varphi_{2} \sim t^{-(d+2) / 2}$ of diffusion in stationary random media, such as the $d$-dimensional Lorentz gas. ${ }^{21}$ Note that the long-time tails are absent in random walks on uniform lattices, where all cumulants $\kappa_{l}$ are vanishing.

The corresponding mean-square displacement for the RBM is given in (4.7). For the RJM we recover the exact results $\left\langle n^{2}\right\rangle_{t}=2 D t$ and $\varphi_{2}(t)=D \delta_{+}(t)$ of Haus et al. ${ }^{7}$

The results (4.4) for the frequency-dependent (modified) Burnett coefficient $U_{2}(z)$ in the RBM are new. The leading long-time tail in its inverse Laplace transform, which is the Burnett correlation function $\varphi_{4}(t)$, behaves as $t^{-3 / 2}$ with a coefficient depending on sixth-order fluctuations. The closely related moment $\left\langle n^{4}\right\rangle_{t}$ is given in (4.8). The HNC diagrams reproduce again the EMA results of Ref. 10 for $\left\langle n^{4}\right\rangle_{t}$, and the EMA values of the coefficients together with the exact ones can be found in Table $I$.

For a discussion of the EMA results it is more instructive to make the following observation. If we denote $U_{0}(z)$ as calculated from the EMA by $W(z)$, then inspection of the structure of the diagrams in Fig. 1 shows that $U(q, z)$ in the EMA is given by $\omega(q) W(z) / D$, so that the response function $\mathscr{F}(q, z)=[z+\omega(q) W(z) / D]^{-1}$. In the EMA all quantities derived from the response function (transport coefficients, correlation functions, and moments of displacement) are completely determined by the frequency-dependent diffusion coefficient $W(z)$. A similar approximation for the Lorentz gas has been proposed by Alder and Alley, ${ }^{27}$ and Ernst and van Beijeren ${ }^{19}$ have shown, using a diagrammatic analysis, that the contributions neglected in Alder and Alley's approximation are of the same type as in the EMA.

The Burnett functions for the RJM behave again very differently from those of the RBM, as can be seen by comparing $U_{2}(z)$ in (4.4) and (4.6). The same result for the dominant contribution to $U_{2}(z)$ in the RJM has also been obtained from the mode-coupling theory, ${ }^{20}$ and from renormalization-group methods. ${ }^{26}$ Also the probability of zero displacement or staying probability $P_{0}(t)$ behaves quite different in RBM and RJM [cf. (4.16) and (4.22)].

The average Green's function determines the spectral properties, which are the same in both models [cf. (3.1) and (3.13)], and also identical to the spectral properties of harmonic chains with random masses or random spring constants. ${ }^{1,35}$ This can be seen by replacing the eigenvalue $\epsilon$ in (2.24) by $\omega^{2}$. In fact, the master equation (1.2) for the RBM describes, after replacement of $\dot{p}_{n}$ by $\ddot{p}_{n}$, the lattice dynamics of a harmonic chain with random spring constants $\left\{w_{n}\right\}$. The eigenvalue problem in lattice dynamics is obtained by the replacement $p_{n}(t)=e^{i \omega t} p_{n}$. Our results (4.17) for the low-frequency behavior of the density of states $\mathscr{N}(\epsilon)$ and for the inverse localization length $\gamma(\epsilon)$ (see Sec. II) are the same as the recent exact result of Nieuwenhuizen, obtained by entirely different methods. ${ }^{35}$

In a separate publication ${ }^{36}$ we have also considered a fluctuation expansion in bare fluctuations $\bar{\delta}_{n}=\left(w_{n}\right.$ $-\langle w\rangle) /\langle w\rangle$ for the RBM, which provides a systematic method to describe the large- $z$ behavior of response and Green's functions. Comparison of the exact results of $U_{0}(z)$ with the corresponding EMA result shows again that the EMA accounts only for the hypernetted-chain diagrams.

The bare fluctuation expansion for the average Green's function gives also a simple method to determine the moments of the average spectral density, $\int_{0}^{\infty} d \epsilon \epsilon^{n} \mathscr{N}(\epsilon)$ in hopping models or in random harmonic chains, in complete agreement with the results of Domb et al. ${ }^{37}$ For the RJM the results have been extended to higher-dimensional systems.

\section{ACKNOWLEDGMENTS}

want to thank T. M. Nieuwenhuizen and $H$. van Beijeren for clarifying discussions.

\section{APPENDIX A}

In this appendix we develop a diagrammatic method for calculating the fluctuation expansion $A(q, z)$ defined in (3.8). The $(l-1)$ th term in the expansion of $g_{0}^{2}(q) A(q, z)$ is apart from a factor $(-z)^{l}$ equal to [cf. (3.7) and (3.8)]

$$
\begin{aligned}
\left\langle g_{0}\left(\Delta g_{0}\right)^{l+1}\right\rangle_{q q^{\prime}}= & g_{0}(q) g_{0}\left(q^{\prime}\right) \\
& \times \sum_{q_{1}, \ldots, q_{l}}\left\langle\Delta_{q q_{1}} \Delta_{q_{1} q_{2}} \cdots \Delta_{q_{l} q^{\prime}}\right\rangle \\
& \times \prod_{i=1}^{l} g_{0}\left(q_{i}\right),
\end{aligned}
$$

where $\Delta_{q q^{\prime}}$ represents the fluctuation

$$
\Delta_{q q^{\prime}}=N^{-1} \sum_{n} \delta_{n} e^{i n\left(q-q^{\prime}\right)}
$$

and the random variables $\delta_{n}=D\left(1 / w_{n}-\langle 1 / w\rangle\right)$ $=D / w_{n}-1$ with $n=0,1, \ldots, N-1$ are distributed independently. It can be represented by diagrams consisting of $(l+2)$ line segments, $a_{i}$, labeled with a wave number $q_{i} \in\left\{q, q_{1}, \ldots, q_{l}, q^{\prime}\right\}$, and $(l+1)$ vertices, $n_{i}$, labeled with a site label $n_{i} \in\left\{n_{1}, \ldots, n_{l+1}\right\}$ and separating the line segments, and of dashed lines $n^{n-\ldots-\ldots} n^{n}$ of connecting vertices with equal site labels.

The contribution of a diagram is determined by the following diagram rules:

(1) a factor $N^{-1} \delta_{n} e^{i n\left(q-q^{\prime}\right)}$ for each $\frac{\text { ' }}{n} q^{\prime}$;

(2) a factor $g_{0}(q)$ for each $\quad q$

(3) a $\delta_{n n^{\prime}}$ for each $n_{0} \ldots$

(4) sum over all internal $q_{i}$;

(5) sum over all $n_{i}$, excluding those terms where unconnected $n_{i}$ are equal;

(6) average over all random variables $\delta_{n}$.

An important statistical concept in the calculation is that $\delta_{n}$ 's are independent random variables. This implies that averages of the form $\left\langle\delta_{n_{1}} \delta_{n_{2}} \delta_{n_{3}} \cdots\right\rangle$ are only nonvanishing if each label occurs at least twice, since $\left\langle\delta_{n}\right\rangle=0$. Therefore, each vertex must be connected with at least one other vertex through a dashed line. If the site label $n$ is connected to $m$ other vertices, it contributes a factor $\left\langle\delta_{n}^{m}\right\rangle=\mu_{m}$, independent of site $n$. 
As an example consider the term of second order in the fluctuation,

$$
\begin{aligned}
\left\langle g_{0}\left(\Delta g_{0}\right)^{2}\right\rangle_{q q^{\prime}} \frac{--\rangle_{1}}{q n_{1} q_{n_{2} q^{\prime}}} & =N^{-2} \sum_{q_{1}} \sum_{n_{1}, n_{2}} e^{i n_{1}\left(q-q_{1}\right)} e^{i n_{2}\left(q_{1}-q^{\prime}\right)} \delta_{n_{1} n_{2}}\left\langle\delta_{n_{1}} \delta_{n_{2}}\right\rangle g_{0}(q) g_{0}\left(q_{1}\right) g_{0}\left(q^{\prime}\right) \\
& =g_{0}(q) g_{0}\left(q^{\prime}\right) \delta_{q q^{\prime}} \mu_{2} h_{1}(z),
\end{aligned}
$$

where (2.3) and (B1) of Appendix B have been used. Observe that matrix elements \langle\rangle$_{q q^{\prime}}$ are diagonal on account of translational invariance.

The third-order term, becomes similarly

$$
\left\langle g_{0}\left(\Delta g_{0}\right)^{3}\right\rangle_{q q^{\prime}} \ldots \bar{r}^{-\cdots}=g_{0}(q) g_{0}\left(q^{\prime}\right) \delta_{q q^{\prime}} \mu_{3} h_{1}^{2}(z) \text {. }
$$

The fourth-order term,

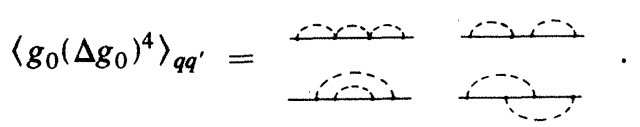

In the first term $n_{1}=n_{2}=n_{3}=n_{4}$ or symbolically (1234), in the next term $n_{1}=n_{2} \neq n_{3}=n_{4}$ or (12)(34) and similarly we have $(14)(23)$ and $(13)(24)$. The first term is simply given by

$$
(1234)=g_{0}(q) g_{0}\left(q^{\prime}\right) \delta_{q q^{\prime}} \mu_{4} h_{1}^{3}(z) \text {. }
$$

Next consider as an example the last term in (A5):

$$
\begin{aligned}
(13)(24)= & g_{0}(q) g_{0}\left(q^{\prime}\right) \mu_{2}^{2} N^{-4} \\
& \times \sum_{q_{1}, q_{2}, q_{3}} \sum_{n_{1} \neq n_{2}} g_{0}\left(q_{1}\right) g_{0}\left(q_{2}\right) g_{0}\left(q_{3}\right) e^{i \varphi}
\end{aligned}
$$

with

$\varphi=n_{1}\left(q-q_{1}+q_{2}-q_{3}\right)+n_{2}\left(q_{1}-q_{2}+q_{3}-q^{\prime}\right)$.

The restricted double sum $\left(n_{1} \neq n_{2}\right)$ in (A7a) is split into an unrestricted double sum minus a single sum $\left(n_{1}=n_{2}\right)$ yielding $\left(N^{2} \delta_{q, q_{1}-q_{2}+q_{3}}-N\right) \delta_{q q^{\prime}}$. Using definition (B5) this term yields

$$
(13)(24)=g_{0}(q) g_{0}\left(q^{\prime}\right) \delta_{q q^{\prime}} \mu_{2}^{2}\left[g_{1}(q, z)-h_{1}^{3}(z)\right] \text {. }
$$

Similarly we find

$$
\begin{aligned}
& (12)(34)=g_{0}(q) g_{0}\left(q^{\prime}\right) \delta_{q q^{\prime}} \mu_{2}^{2}\left[g_{0}(q) h_{1}^{2}(z)-h_{1}^{3}(z)\right], \\
& (14)(23)=g_{0}(q) g_{0}\left(q^{\prime}\right) \delta_{q q^{\prime}} \mu_{2}^{2}\left[h_{1}(z) h_{2}(z)-h_{1}^{3}(z)\right] .
\end{aligned}
$$

Using the cumulants (3.16) the terms (A6), (A8), and (A9) can be combined into

$$
\begin{aligned}
\left\langle g_{0}\left(\Delta g_{0}\right)^{4}\right\rangle_{q q^{\prime}}= & g_{0}(q) g_{0}\left(q^{\prime}\right) \delta_{q q^{\prime}} \\
& \times\left[\kappa_{4} h_{1}^{3}+\kappa_{2}^{2}\left(g_{0} h_{1}^{2}+h_{1} h_{2}+g_{1}\right)\right] .
\end{aligned}
$$

We may therefore modify the diagram rules and assign a value $\kappa_{4} h_{1}^{3}$ to the first diagram in (A5) with four connected vertices, and values $\kappa_{2}^{2} g_{0} h_{1}^{2}, \kappa_{2}^{2} h_{1} h_{2}$, and $\kappa_{2}^{2} g_{1}$ to the second, third, and fourth diagrams, respectively, with two pairs of connected vertices. In general we assign to each set of $j$ connected vertices a weight, given by the $j$ th cumulant $\kappa_{j}$.

The number of independent $q_{i}$ variables in the modified diagrams can be determined by observing that the total wave number is conserved at each vertex, i.e., if we assign wave numbers to solid lines and dashed lines, the sum of incoming $q$ 's equals that of outgoing $q$ 's. Consequently, each term in \langle\rangle$_{q q^{\prime}}$ is proportional to $\delta_{q q^{\prime}}$.

The diagram contributions are now calculated according to the modified diagram rules:

(1) label external lines with wave vector $q$; label internal lines such that the sum of incoming wave numbers equals the sum of outgoing wave numbers;

(2) a factor $g_{0}(q)$ for line segment $q$;

(3) sum over all independent internal $q$ 's with a weight $N^{-1}$

(4) a factor $\prod_{j=2}^{\infty}\left(\kappa_{j}\right)^{m_{j}}$ for a diagram with $m_{j}$ $(j=2,3, \ldots)$ sets of $j$ connected vertices.

Next we consider fifth- and sixth-order fluctuations. The fifth-order terms are symbolically denoted as (12345) (one such term) and (12)(345), (13)(245), (14)(235), (15)(234), (123)(45), (124)(35), (125)(34), (134)(25), $(135)(24),(145)(23)$ (ten such terms), or by corresponding diagrams (see Fig. 1), where all diagrams are listed as well as the corresponding analytic expressions and the multiplicity of each diagram. The subtracted terms in the 10 terms of type $(\cdots)(\cdot)$ contain factors $\mu_{3} \mu_{2} h_{1}^{4}(z)$, that can be combined with the contribution $\mu_{5} h_{1}^{4}(z)$ from (12345) to yield the modified diagram, containing cumulants instead of moments $\mu_{k}$, represented by

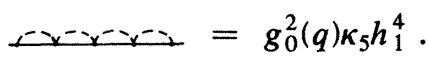

The remaining contribution of $(\cdots)(\cdot \cdot)$ can be calculated directly from the modified diagram rules. As an example consider (135)(24),

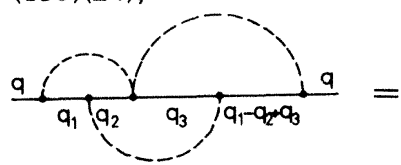

$$
\kappa_{2} \kappa_{3} g_{0}^{2}(q) N^{-3} \sum_{q_{1}} g_{0}\left(q_{1}\right) \sum_{q_{2}, q_{3}} g_{0}\left(q_{2}\right) g_{0}\left(q_{3}\right) g_{0}\left(q_{1}-q_{2}+q_{3}\right)=\kappa_{2} \kappa_{3} g_{0}^{2}(q) N^{-1} \sum_{q_{1}} g_{0}\left(q_{1}\right) g_{1}\left(q_{1}, z\right)=\kappa_{2} \kappa_{3} g_{0}^{2}(q) \tilde{h}_{1}(z) \text {, }
$$

where the definitions (B5) and (B9) of Appendix B have been used. In a similar way we calculate the remaining terms. The result is 


$$
\left\langle g_{0}\left(\Delta g_{0}\right)^{5}\right\rangle_{q q}=g_{0}^{2}(q)\left\{\kappa_{5} h_{1}^{4}(z)+\kappa_{2} \kappa_{3}\left[2 g_{0}(q) h_{1}^{3}(z)+3 h_{1}^{2}(z) h_{2}(z)+4 h_{1}(z) g_{1}(q, z)+\widetilde{h}_{1}(z)\right]\right\} .
$$

The function $h_{2}(z)$ is defined in (B1).

The calculation of the sixth-order term is much more involved. We shall only illustrate how the subtracted terms resulting from the restricted $n_{i}$ summations may be combined with other terms to yield the modified diagrams. Consider the following terms calculated according to the original diagram rules:

$$
\begin{aligned}
& (123456)=\mu_{6} g_{0}^{2} h_{1}^{5}, \\
& (12)(3456)=\mu_{2} \mu_{4} g_{0}^{2}\left(g_{0} h_{1}^{4}-h_{1}^{5}\right), \\
& (123)(456)=\mu_{3}^{2} g_{0}^{2}\left(g_{0} h_{1}^{4}-h_{1}^{5}\right), \\
& (12)(34)(56)=\mu_{2}^{3} g_{0}^{2}\left(g_{0}^{2} h_{1}^{3}-2 g_{0} h_{1}^{4}-h_{1}^{3} h_{2}+2 h_{1}^{5}\right) .
\end{aligned}
$$

With the use of the definition (3.16) of the cumulant $\kappa_{6}$ the last terms on each line, proportional to $h_{1}^{5}$, can be combined into a modified diagram (123456) with a value $\kappa_{6} h_{0}^{2} h_{1}^{5}$, as there are 15 terms of type $(\cdot)(\cdots), 10$ of type $(\cdots)(\cdots)$, and 15 of type $(\cdot)(\cdot)(\cdot)$. A more detailed comparison of terms enables us to combine $\mu_{2} \mu_{4} g_{0}^{3} h_{1}^{4}$ with those of type $(\cdot)(\cdot)(\cdot)$ to obtain the modified diagram (12)(3456) with a value $\kappa_{2} \kappa_{4} g_{0}^{3} h_{1}^{4}$. In this way one finds that the first term on each line of (A14) represents the contribution according to the modified diagram rules, provided all moments $\mu_{l}$ are replaced by cumulants $\kappa_{l}$. As an example we give the modified diagram contribution from (15)(23)(46),

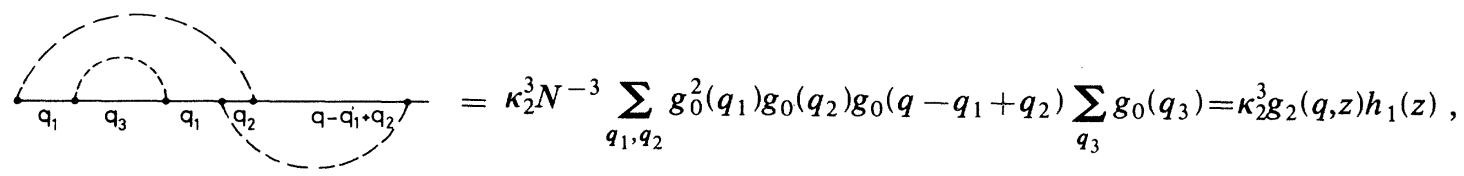

where (B5) has been used. The total contribution of the sixth-order fluctuation terms gives finally (see also Fig. 1):

$$
\begin{aligned}
\left\langle g_{0}\left(\Delta g_{0}\right)^{6}\right\rangle= & g_{0}^{2} \kappa_{2}^{3}\left(g_{0}^{2} h_{1}^{3}+2 g_{0} h_{1} g_{1}+2 g_{0} h_{1}^{2} h_{2}+3 h_{1} g_{2}+4 g_{3}+h_{1}^{2} h_{3}+h_{1} h_{2}^{2}+\widetilde{h}_{2}\right) \\
& +g_{0}^{2} \kappa_{3}^{2}\left(g_{0} h_{1}^{4}+4 h_{1}^{2} g_{1}+2 h_{1}^{2} h_{2}+2 h_{1} \widetilde{h}_{1}+g_{4}\right)+g_{0}^{2} \kappa_{2} \kappa_{4}\left(2 g_{0} h_{1}^{4}+6 h_{1} g_{1}^{2}+4 h_{1}^{3} h_{2}+3 h_{1}{\widetilde{h_{1}}}_{1}\right)+g_{0}^{2} \kappa_{6} h_{1}^{5} .
\end{aligned}
$$

All functions have been defined in (B1), (B5), and (B9), except

$$
\begin{array}{r}
g_{4}(q, z)=N^{-4} \sum_{q_{1}, \ldots, q_{4}} g_{0}\left(q_{1}\right) g_{0}\left(q_{2}\right) g_{0}\left(q_{3}\right) g_{0}\left(q_{4}\right) \\
\times g_{0}\left(q-q_{1}+q_{2}-q_{3}+q_{4}\right) .
\end{array}
$$

The notation is systematic in the sense that all functions $g_{i}(q, z)$ with $i=1,2, \ldots$, represent contributions from "self-energy" diagrams that depend on the external wave number $q$, whereas the remaining contributions $h_{i}(z)$ and $\widetilde{h_{i}}(z)(i=1,2, \ldots$,$) are independent of q$. The simplest $q-$ dependent self-energy diagram is the fourth one in (A5) with a contribution $\kappa_{2}^{2} g_{1}(q, z)$. Also note that the diagrams whose contribution does not contain the function $g_{n}$ $(n \geq 1)$ or $\widetilde{h}_{n}(h \geq 1)$ are built as series-parallel circuits of the very first diagram. These diagrams will be referred to as hypernetted-chain diagrams. Their contributions can be expressed as products (of powers) of $h_{1}, h_{2}, h_{3}, \ldots$, where $h_{n+1}=(-1)^{n} h_{1}^{(n)} / n !(n \geq 0)$ [see (B1) and (B2)] and the superscript on $h_{1}$ denotes the $n$th derivative with respect to $z$.

\section{APPENDIX B}

The calculation of the separate terms in the fluctuation expansion presented in Appendix $A$ is in fact independent of the number of dimensions. The behavior of these terms for small $z$ and small $q$ will strongly depend on the dimensionality of the system.

In this appendix we define the functions appearing in
Appendix A and in the results quoted in Sec. III, and we will calculate them for the one-dimensional case. When possible we give exact expressions, otherwise we determine the dominant small-z behavior with $q=\kappa \sqrt{z}$ and $\kappa$ kept fixed, which is sufficient for our applications.

In the remaining part of this appendix we present a power counting argument to estimate the leading small-z behavior of all terms in the diagrammatic expansion. These arguments are given for the one-dimensional case, but can be extended to general dimensions in a straightforward manner.

We define

$$
h_{n}(z)=N^{-1} \sum_{q} g_{0}^{n}(q), n=1,2, \ldots,
$$

with $g_{0}(q)=[z+\omega(q)]^{-1}$ and $\omega(q)=2 D(1-\cos q)$. From (B1) we have

$$
h_{n}(z)=(1-n)^{-1} h_{n-1}^{\prime}(z), \quad n=2,3, \ldots .
$$

The prime denotes differentiation with respect to $z$. On account of (B2) all these functions can be derived from $h_{1}(z)$. In the thermodynamic limit $(N \rightarrow \infty)$ the sums $N^{-1} \sum_{q} \cdots$ with $q \in 1 \mathrm{BZ}$ may be replaced by integrals $(2 \pi)^{-1} \int_{-\pi}^{\pi} d q \cdots$, and the function $h_{1}(z)$ reduces to an elementary integral:

$$
\begin{aligned}
h_{1}(z) & =(2 \pi)^{-1} \int_{-\pi}^{\pi} d q[z+2 D(1-\cos q)]^{-1} \\
& =[z(z+4 D)]^{-1 / 2} .
\end{aligned}
$$

A related set of functions is 


$$
k_{n}(z)=N^{-1} \sum_{q} \omega(q) g_{0}^{n}(q), n=1,2, \ldots
$$

Because (B2) is also valid for $k_{n}$ and $k_{1}(z)=1-z h_{1}(z)$ all $k$ functions can be derived from $h_{1}(z)$.

The next set of functions to be defined is $g_{n}(q, z)$ $(n=1,2,3)$, with

$$
\begin{aligned}
g_{1}(q, z) & =N^{-2} \sum_{q_{1}, q_{2}} g_{0}\left(q_{1}\right) g_{0}\left(q_{2}\right) g_{0}\left(q-q_{1}+q_{2}\right) \\
g_{2}(q, z) & =N^{-2} \sum_{q_{1}, q_{2}} g_{0}^{2}\left(q_{1}\right) g_{0}\left(q_{2}\right) g_{0}\left(q-q_{1}+q_{2}\right) \\
& =-\frac{1}{3} \frac{d}{d z} g_{1}(q, z)
\end{aligned}
$$

and

$$
\begin{aligned}
g_{3}(q, z)=N^{-3} \sum_{q_{1}, q_{2}, q_{3}} & g_{0}\left(q_{1}\right) g_{0}\left(q_{2}\right) g_{0}\left(q_{3}\right) \\
& \times g_{0}\left(q-q_{1}+q_{3}\right) g_{0}\left(q-q_{2}+q_{3}\right) .
\end{aligned}
$$

The function $g_{1}(q, z)$ can be evaluated exactly by transforming the integration variables according to $w_{k}=\exp \left(i q_{k}\right)(k=1,2)$ and $w=\exp (i q)$. Then $g_{1}(q, z)$ is a double integral over the unit circles $c_{1}$ in the complex $w_{1}$ and $w_{2}$ plane:

$$
g_{1}(q, z)=(-D)^{3}(2 \pi i)^{-2} \oint_{c_{1}} \oint_{c_{1}} d w_{1} d w_{2} \frac{w w_{1} w_{2}\left[\left(w w_{1} w_{2}-e^{\alpha}\right)\left(w w_{1} w_{2}-e^{-\alpha}\right)\right]^{-1}}{\left(w_{1}-e^{\alpha}\right)\left(w_{1}-e^{-\alpha}\right)\left(w_{2}-e^{\alpha}\right)\left(w_{2}-e^{-\alpha}\right)}
$$

where we have defined $e^{\alpha}+e^{-\alpha}=2 \cosh \alpha \equiv 2+z / D$.

The integration can be performed using complex contour integration, yielding

$g_{1}(q, z)=\left(8 D^{3}\right)^{-1} \cosh (3 \alpha)(\sinh \alpha)^{-3}[\cosh (3 \alpha)-\cos q]^{-1}$.

In our calculation we only need the small-z and $-q$ behavior (with $q=\kappa \sqrt{z}$ and $\kappa$ fixed). The dominant contribution for $q, z \rightarrow 0$ is

$$
\begin{aligned}
& g_{1}(q, z) \simeq 3(4 D z)^{-1}\left(9 z+D q^{2}\right)^{-1} \\
& g_{2}(q, z) \simeq\left(4 D z^{2}\right)^{-1}\left(18 z+D q^{2}\right)\left(9 z+D q^{2}\right)^{-2}
\end{aligned}
$$

This dominant behavior can also be obtained directly by substituting $q_{k}=x_{k}(z / D)^{1 / 2}$ in the original $q$ integral and letting the integration boundaries $\pm \pi(D / z)^{1 / 2}$ tend to infinity. The remaining integrals can be performed using complex contour integration. This method gives for $g_{3}(q, z)(q, z \rightarrow 0)$

$g_{3}(q, z) \simeq\left(2^{5} D^{3 / 2} z^{5 / 2}\right)^{-1}\left(45 z+D q^{2}\right)\left(9 z+D q^{2}\right)^{-2}$.

We further need $\widetilde{h}_{n}(z)(n=1,2)$ :

$$
\tilde{h}_{n}(z)=N^{-1} \sum_{q} g_{0}^{n}(q) g_{1}(q, z)
$$

which can be evaluated in the same way as sketched for $g_{1}(q, z)$ and gives

$$
\begin{aligned}
& \widetilde{h}_{1}(z)=\left(2^{5} D^{4}\right)^{-1} \sinh (4 \alpha)(\sinh \alpha)^{-4}[\sinh (2 \alpha)]^{-2} \simeq 2^{-5} D^{-3 / 2} z^{-5 / 2} \text { as } z \rightarrow 0, \\
& \tilde{h}_{2}(z)=\left(2^{7} D^{5}\right)^{-1}\left\{(\sinh \alpha)^{2}+[\sinh (3 \alpha)]^{2}\right\}(\sinh \alpha)^{-7}[\sinh (2 \alpha)]^{-2} \simeq 5 \times 2^{-8} D^{-3 / 2} z^{-7 / 2} \text { as } z \rightarrow 0 .
\end{aligned}
$$

Again the dominant contribution for $z \rightarrow 0$ can be obtained more easily by the method described below (B7).

The last set of functions needed is defined as

$$
\begin{array}{ll}
\bar{f}_{n}(z)=N^{-1} \sum_{q} \omega(q) g_{0}(q) g_{n}(q, z), & n=1, \\
f_{n}(z)=N^{-1} \sum_{q} \omega(q) g_{0}^{2}(q) g_{n}(q, z), & n=1,2,3, \\
\tilde{f}_{n}(z)=N^{-1} \sum_{q} \omega(q) g_{0}^{3}(q) g_{n}(q, z), & n=1 .
\end{array}
$$

Only for $\bar{f}_{1}$ and $f_{1}$ we have an exact expression; for the others the dominant behavior for $z \rightarrow 0$ is evaluated as before:

$$
\begin{aligned}
& \bar{f}_{1}(z)=\left(2^{4} D^{3}\right)^{-1}[\sinh (3 \alpha)-\sinh (2 \alpha)-\sinh \alpha](\sinh \alpha)^{-5}[\sinh (2 \alpha)]^{-1} \simeq 3 \times 2^{-5} D^{-3 / 2} z^{-3 / 2} \text { as } z \rightarrow 0, \\
& f_{1}(z)=\tilde{h}_{1}(z)-z \widetilde{h}_{2}(z) \approx 3 \times 2^{-8} D^{-3 / 2} z^{-5 / 2} \text { as } z \rightarrow 0, \\
& f_{2}(z) \simeq 7 \times 2^{-10} D^{-3 / 2} z^{-7 / 2} \text { as } z \rightarrow 0, \\
& f_{3}(z) \simeq 2^{-9} D^{-2} z^{-4} \text { as } z \rightarrow 0, \\
& \widetilde{f}_{1}(z) \simeq 9 \times 2^{-11} D^{-3 / 2} z^{-7 / 2} \text { as } z \rightarrow 0 .
\end{aligned}
$$

In the remaining part of this appendix we shall demonstrate that the fluctuation expansion [(3.7) and (3.8)] is a sys- 
tematic one for small $z$ with $q=\kappa \sqrt{z}$ and $\kappa$ fixed. The small- $z,-q$ behavior of the functions $g_{n}(q, z), h_{n}(z)$, and $\widetilde{h_{n}}(z)$ with $n=1,2,3$ has already been determined above. However, this behavior can also be obtained from simple power counting arguments that can be applied to arbitrary diagrams. As an illustration consider $g_{3}(q, z)$ in (B5) where $q=\kappa(z / D)^{1 / 2}$. In the thermodynamic limit $N^{-1} \sum_{q \in 1 \mathrm{BZ}} \cdots$ can be replaced by $(2 \pi)^{-1} \int_{-\pi}^{\pi} d q \cdots$. The most dominant contribution to this integral comes from $q$ values of order $\sqrt{z}$. Thus we change variables $q_{i}=x_{i}(z / D)^{1 / 2}$ so that $g_{3}(q, z)$ yields to dominant order for small $z$

$$
g_{3}(q, z)=(z / D)^{3 / 2} z^{-5}(2 \pi)^{-3} \int_{-\infty}^{\infty} \int_{-\infty}^{\infty} \int_{-\infty}^{\infty} d x d y d z \frac{\left\{\left[1+(\kappa-x+z)^{2}\right]\left[1+(\kappa-y+z)^{2}\right]\right\}^{-1}}{\left(1+x^{2}\right)\left(1+y^{2}\right)\left(1+z^{2}\right)}
$$

In general, the power counting argument to estimate the small- $z$ behavior of diagrams gives a factor $\sqrt{z}$ for each independent internal $q$ summation and a factor $z^{-1}$ for each $g_{0}$. One readily verifies that this method applied to integrals of Appendix $B$, which are divergent for $z \rightarrow 0$, predicts the correct leading $z$ dependence. Thus we have the following small- $z$ estimates:

$$
\begin{aligned}
& \left\langle\left(z \Delta g_{0}\right)^{2}\right\rangle \simeq \kappa_{2} O\left(z^{1 / 2}\right), \\
& \left\langle\left(z \Delta g_{0}\right)^{3}\right\rangle \simeq \kappa_{3} O(z), \\
& \left\langle\left(z \Delta g_{0}\right)^{4}\right\rangle \simeq \kappa_{2}^{2} O(z)+\kappa_{4} O\left(z^{3 / 2}\right),
\end{aligned}
$$

$$
\begin{aligned}
& \left\langle\left(z \Delta g_{0}\right)^{5}\right\rangle \simeq \kappa_{2} \kappa_{3} O\left(z^{3 / 2}\right)+\kappa_{5} O\left(z^{2}\right), \\
& \left\langle\left(z \Delta g_{0}\right)^{6}\right\rangle \simeq \kappa_{2}^{3} O\left(z^{3 / 2}\right)+\left(\kappa_{4} \kappa_{2}+\kappa_{3}^{2}\right) O\left(z^{2}\right)+\kappa_{6} O\left(z^{5 / 2}\right) \\
& \left\langle\left(z \Delta g_{0}\right)^{2 l-1}\right\rangle \simeq \kappa_{3} \kappa_{2}^{l-2} O\left(z^{l / 2}\right) \\
& \left\langle\left(z \Delta g_{0}\right)^{2 l}\right\rangle \simeq \kappa_{2}^{l} O\left(z^{l / 2}\right) .
\end{aligned}
$$

These estimates show that the fluctuation expansion (3.8) for $g_{0}(q) A(q, z)$ has a dominant behavior for small $z$ with $q \simeq \kappa \sqrt{z}$ of order $z^{-3 / 2}$. In order to calculate this term exactly to order $z^{-1 / 2}$ included fifth- and sixth-order fluctuation formulas have to be included.
${ }^{1}$ S. Alexander, J. Bernasconi, W. R. Schneider, and R. Orbach, Rev. Mod. Phys. 53, 175 (1981).

2J. Machta, Phys. Rev. B 24, 5260 (1981).

${ }^{3}$ T. Odagaki and M. Lax, Phys. Rev. B 24, 5284 (1981).

4I. Webman, Phys. Rev. Lett. 47, 1496 (1981).

${ }^{5}$ Proceedings of the Conference on Macroscopic Properties of Disordered Media, edited by E. Burridge, S. Childress, and G. Papanicolau (Springer, New York, 1982).

${ }^{6}$ T. Odagaki and M. Lax, Phys. Rev. B 25, 2301 (1982); 25, 2307 (1982).

7J. W. Haus, K. W. Kehr, and J. Lyklema, Phys. Rev. B $\underline{25}$, 2905 (1982).

8 J. W. Haus, K. W. Kehr, and K. Kitahara, Phys. Rev. B $\underline{25}$, 4918 (1982)

${ }^{9}$ M. J. Stephen and R. Kariotis, Phys. Rev. B 26, 2917 (1982).

${ }^{10}$ I. Webman and J. Klafter, Phys. Rev. B 26, 5950 (1982).

${ }^{11}$ B. Derrida and Y. Pomeau, Phys. Rev. Lett. 48, 627 (1982).

${ }^{12}$ R. Zwanzig, J. Stat. Phys. 28, 127 (1982).

${ }^{13}$ V. V. Anshelevich and A. V. Vologodskii, J. Phys. A 15,185 (1982).

${ }^{14}$ V. Halpern, J. Phys. C 15, L827 (1982).

${ }^{15}$ H. van Beijeren, Rev. Mod. Phys. 54, 195 (1982).

${ }^{16}$ V. M. Kenkre, J. Machta, and J. Bernasconi, in Proceedings of a Symposium on Random Walks and Their Applications to the Physical and Biological Sciences [J. Stat. Phys. 30, 293 (1983)].

17J. W. Haus, K. W. Kehr, and K. Kitahara, Z. Phys. B $\underline{50}, 161$ (1983).

${ }^{18}$ B. Derrida, J. Stat. Phys. 31, 433 (1983).

${ }^{19}$ M. H. Ernst and H. van Beijeren, J. Stat. Phys. 26, 1 (1981).

${ }^{20}$ M. H. Ernst, J. Machta, J. R. Dorfman, and H. van Beijeren,
J. Stat. Phys. (in press).

${ }^{21}$ M. H. Ernst and A. Weyland, Phys. Lett. 34A, 39 (1971).

22J. M. J. v. Leeuwen, J. Groeneveld, and J. de Boer, Physica $\underline{25}$, 792 (1959).

${ }^{23} \mathrm{G}$. Stell, in The Equilibrium Theory of Classical Fluids, edited by H. L. Frisch and J. L. Lebowitz (Benjamin, New York, 1964).

${ }^{24}$ T. M. Nieuwenhuizen, Physica A (in press).

${ }^{25}$ K. W. Kehr, D. Richter, and R. H. Swendsen, J. Phys. F $\underline{8}$, 433 (1978).

26J. Machta, in Proceedings of a Symposium on Random Walks and Their Applications to the Physical and Biological Sciences, Ref. 16.

${ }^{27}$ B. J. Alder and W. E. Alley, J. Stat. Phys. 19, 341 (1978).

${ }^{28}$ D. J. Thouless, J. Phys. C $\underline{5}, 77$ (1972).

${ }^{29}$ H. Matsuda and K. Ishii, Prog. Theor. Phys. Suppl. 45, 56 (1970).

${ }^{30}$ H. Fürstenberg, Trans. Am. Math. Soc. 108, 377 (1963).

${ }^{31}$ T. M. Nieuwenhuizen, Physica 113A, 173 (1982).

32T. Morita, J. Math. Phys. 12, 1744 (1971).

${ }^{33}$ I. Gradshteyn and I. Ryzhik, Tables of Integrals, Series and Products (Academic, New York, 1980).

${ }^{34}$ T. Morita and T. Horiguchi, J. Math. Phys. 12, 981 (1971); 12,986 (1971).

${ }^{35}$ T. M. Nieuwenhuizen, Ph.D. dissertation, State University of Utrecht, 1983 (unpublished).

36P. J. H. Denteneer and M. H. Ernst, J. Phys. C 16, L961 (1983).

${ }^{37}$ C. Domb, A. A. Maradudin, E. W. Montroll, and G. H. Weiss, Phys. Rev. 115, 18 (1959). 